\title{
Chitin, Chitin Oligosaccharide, and Chitin Disaccharide Metabolism of Escherichia coli Revisited: Reassignment of the Roles of ChiA, ChbR, ChbF, and ChbG
}

\author{
Axel Walter Simon Friz Christoph Mayer \\ Interfaculty Institute of Microbiology and Infection Medicine, Organismic Interactions/Glycobiology, Eberhard Karls \\ Universität Tübingen, Tübingen, Germany
}

\section{Keywords \\ Chitin - Peptidoglycan - Chitobiose - chb operon · \\ Diacetylchitobiose 6 '-phosphate monodeacetylase . \\ 6-Phospho- $\beta$-glucosaminidase - Family 4 glycosidase}

\begin{abstract}
Escherichia coli is unable to grow on polymeric and oligomeric chitin, but grows on chitin disaccharide (GICNAcGlcNAc; $N, N^{\prime}$-diacetylchitobiose) and chitin trisaccharide (GlcNAc-GlcNAc-GlcNAc; $N, N^{\prime}, N^{\prime \prime}$-triacetylchitotriose) via expression of the chb operon (chbBCARFG). The phosphotransferase system (PTS) transporter ChbBCA facilitates transport of both saccharides across the inner membrane and their concomitant phosphorylation at the non-reducing end, intracellularly yielding GlcNAc 6-phosphate-GIcNAc (GlcNAc6P-GlcNAc) and GlcNAc6P-GlcNAc-GlcNAc, respectively. We revisited the intracellular catabolism of the PTS products, thereby correcting the reported functions of the 6-phospho-glycosidase ChbF, the monodeacetylase ChbG, and the transcriptional regulator ChbR. Intracellular accumulation of glucosamine 6P-GlcNAc (GlcN6P-GlcNAc) and GlcN6P-GlcNAc-GlcNAc in a chbF mutant unraveled a role for ChbG as a monodeacetylase that removes the $\mathrm{N}$-acetyl group at the non-reducing end. Consequently, GlcN6P- but not GlcNAc6P-containing saccharides likely function as co-
\end{abstract}

karger@karger.com www.karger.com/mip

Karger $\stackrel{\text { ' }}{5}$
(C) 2021 The Author(s)

Published by S. Karger AG, Basel

This is an Open Access article licensed under the Creative Commons Attribution-NonCommercial-4.0 International License (CC BY-NC) (http://www.karger.com/Services/OpenAccessLicense), applicable to the online version of the article only. Usage and distribution for commercial purposes requires written permission. activators of ChbR. Furthermore, ChbF removed the GlcN6P from the non-reducing terminus of the former saccharides, thereby degrading the inducers of the chb operon and facilitating growth on the saccharides. Consequently, ChbF was unable to hydrolyze GIcNAc6P-residues from the nonreducing end, contrary to previous assumptions but in agreement with structural modeling data and with the unusual catalytic mechanism of the family 4 of glycosidases, to which ChbF belongs. We also refuted the assumption that ChiA is a bifunctional endochitinase/lysozyme ChiA, and show that it is unable to degrade peptidoglycans but acts as a bona fide chitinase in vitro and in vivo, enabling growth of E. coli on chitin oligosaccharides when ectopically expressed. Overall, this study revises our understanding of the chitin, chitin oligosaccharide, and chitin disaccharide metabolism of E. coli.

(C) 2021 The Author(s).

Published by S. Karger AG, Basel

\section{Introduction}

Chitin is a homopolymer composed of $\beta-1,4$ linked $N$ acetylglucosamine (GlcNAc) found in the cell walls of fungi, exoskeletons of crustaceans and insects, as well as the shells of molluscs. It is the most abundant nitrogen- 
containing polysaccharide on earth, with an annual turnover rate of $10^{11}$ tons, therefore playing a central role in the global nitrogen and carbon cycle [Tharanathan and Kittur, 2003]. Degradation of the recalcitrant chitin biopolymer is achieved by chitinolytic fungi as well as bacteria, e.g., gram-positive bacteria of the genus Bacillus or Streptomyces, and gram-negative bacteria of the genus Serratia, Aeromonas, or Vibrio, which play an important role in chitin recycling in soil and aquatic (marine) ecosystems [Blokesch, 2012; Hamid et al., 2013; Adams et al., 2019; Wucher et al., 2019]. These organisms secrete various forms of chitinases and $\mathrm{N}$-acetylglucosaminidases that fragment polymeric chitin into shorter chitin oligosaccharides, $\quad N, N^{\prime}, N^{\prime \prime}$-triacetylchitotriose (GlcNAcGlcNAc-GlcNAc, triacetyl-chitotriose), N,N'-diacetylchitobiose (GlcNAc-GlcNAc, diacetyl-chitobiose), and GlcNAc [Itoh and Kimoto, 2019]. Furthermore, they possess uptake and catabolic systems to grow on chitin and chitin oligosaccharides [Hamid et al., 2013; Itoh and Kimoto, 2019].

Escherichia coli $\mathrm{K} 12$ is unable to grow on chitin and chitin oligosaccharides, despite possessing a functional chitinase (ChiA/YheB) and an outer membrane porin that facilitates chitin oligosaccharide uptake (ChiP) [Francetic et al., 2000a; Soysa and Suginta, 2016]. Under normal laboratory growth conditions, the expression of ChiA and its secretion via the type 2 secretion system is silenced, requiring a mutation in the $h n s$ gene for activation [Francetic et al., 2000a, b]. As ChiA contains a native signal sequence, in an hns background, the chitinase is expressed and partially secreted to the periplasm via the Sec system and to the extracellular space via the type 2 secretome [Francetic et al., 2000a, b]. We became interested in the cryptic ChiA, since it had been described as a bifunctional enzyme with endo-chitinase and lysozyme activity, an assumption that, however, solely relied on the enzyme's ability to cleave the artificial lysozyme substrate ethylene glycol-chitin [Francetic et al., 2000a]. We thus aimed to re-examine the function of ChiA and chitin metabolism in E. coli.

Growth of E. coli on diacetyl-chitobiose and triacetylchitotriose depends on the chitobiose operon chb$B C A R F G$. This operon was initially identified as the cryptic cel (cellobiose utilization) operon, which requires secondary mutations in order to metabolize $\beta$-glycosides such as cellobiose, arbutin, and salicin [Parker and Hall, 1990]. Later it was shown that the operon is not cryptic but induced by diacetyl-chitobiose, an end product of chitin degradation, and therefore renamed to chb [Keyhani and Roseman, 1997]. Encoded by the chbBCARFG operon are components of a phosphotransferase system (PTS) transporter (ChbBCA; EIIB, EIIC, and EIIA) involved in both, the transport of diacetyl-chitobiose and triacetyl-chitotriose and their simultaneous phosphorylation at the 6-hydroxyl group of the GlcNAc at the nonreducing end [Keyhani et al., 2000a, b, c]. Further encoded by this operon is a transcriptional repressor/activator (ChbR), repressing chb transcription by default and acting as an activator upon effector binding [Plumbridge and Pellegrini, 2004]. The chb operon is also subject to catabolite repression and is repressed by NagC, the transcriptional regulator of the GlcNAc catabolism, which is derepressed upon GlcNAc6P binding [Plumbridge and Pellegrini, 2004]. The enzymes ChbG and ChbF are involved in the intracellular processing of the phosphorylated diacetyl-chitobiose (diacetyl-chitobiose-P) and triacetyl-chitotriose (triacetyl-chitotriose-P). ChbG was identified as a monodeacetylase that is essential for growth on diacetyl-chitobiose and triacetyl-chitotriose but is dispensable for growth on cellobiose and chitobiose (chitosan dimer; GlcN- $\beta-1,4-G l c N$ ) [Verma and Mahadevan, 2012]. Verma and Mahadevan [2012] further showed that activation of the $c h b$ promoter by the regulatory protein $\mathrm{ChbR}$ is dependent on $\mathrm{ChbG}$, suggesting that deacetylation of diacetyl-chitobiose-P and triacetyl-chitotriose- $\mathrm{P}$ is necessary for their function as co-activators of ChbR. The authors proposed that ChbG removes the $\mathrm{N}$-acetyl group from the reducing end, yielding GlcNAc6P-GlcN and GlcNAc6P-GlcNAc-GlcN, which may act as the inducers of the chb operon upon ChbR binding [Verma and Mahadevan, 2012]. Subsequent cleavage of these saccharides would yield GlcNAc6P, which upon binding to NagC could relieve repression of the chb operon. We questioned these assumptions, because the ChbF protein, which is responsible for the hydrolysis of the ChbG products, monoacetyl-chitobiose-P and diacetyl-chitotriose- $\mathrm{P}$, was initially characterized as cellobiose-6-phosphate (Glc6P-Glc) hydrolase (named CelF) [Parker and Hall, 1990; Thompson et al., 1999]. Since ChbF cleaves Glc6P-Glc and other 6-phospho-glucosides (e.g., arbutin-P and salicin-P) and as well as chitobiose-P (GlcN6P-GlcN), we suggest that ChbF likely cleaves GlcN6P-glycosides (GlcN6P-GlcNAc and GlcN6P-GlcNAc-GlcNAc), rather than GlcNAc6P-glycosides. $\mathrm{ChbF}$ is assigned to the family 4 of glycosidases (GH4). The proposed mechanism for this class of enzymes is very distinct from that of classical glycosidases, which operate via nucleophilic substitution-mechanisms. GH4 glycosidases instead depend on a divalent metal ion and $\mathrm{NAD}^{+}$as cofactors and catalyze an oxidation/reduc- 


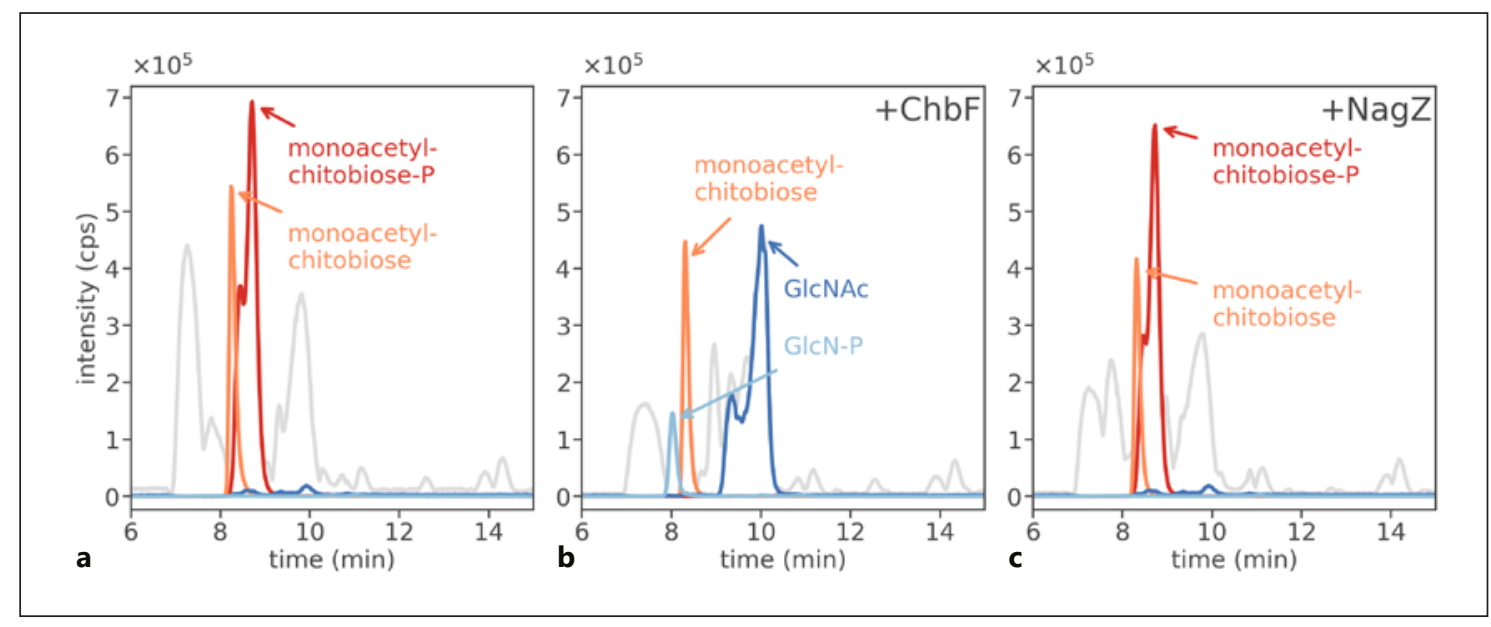

Fig. 1. Accumulation of monoacetyl-chitobiose-P and monoacetyl-chitobiose in $\Delta c h b F:: k a n$ cells and characterization of the ChbF and NagZ products. The cytosolic fraction of E. coli $\Delta c h b F:: k a n$ cells, grown in M9 CAA minimal medium supplemented with diacetyl-chitobiose, was extracted and analyzed by LC-MS. a Accumulation products with masses $(\mathrm{m} / \mathrm{z})$ corresponding to monoacetyl-chitobiose- $\mathrm{P}(\mathrm{M}+\mathrm{H})^{+} \mathrm{m} / \mathrm{z}=463.0976$ (red) and monoacetylchitobiose $(\mathrm{M}+\mathrm{H})^{+} \mathrm{m} / \mathrm{z}=383.1369$ (orange) were detected. b Upon treatment with $\mathrm{ChbF}(+\mathrm{ChbF})$, the monoacetyl-chitobiose- $\mathrm{P}(\mathrm{M}+\mathrm{H})^{+} \mathrm{m} / \mathrm{z}=463.0976$ (red) completely disappeared, while

tion of the hydroxyl group at C3 of the sugar at the nonreducing end of a disaccharide. Formation of an intermediate carbonyl at $\mathrm{C} 3$ renders the $\mathrm{C} 2$ proton more acidic and thereby facilitates the elimination of the glycosidic oxygen, that is, the cleavage of the glycosidic bond [Rajan et al., 2004; Yip et al., 2004, 2007; Yip and Withers, 2006a, b]. Since ChbF operates by an mechanism that involves reactions at $\mathrm{C} 3$ and $\mathrm{C} 2$, the $\mathrm{N}$-acetyl group at C2 likely interferes with this mechanism [Thompson et al., 1999]. Here we revised the function of $\mathrm{ChbF}$ as well as of $\mathrm{ChbG}$ and unraveled the nature of the effector molecules of the transcriptional regulator ChbR. Furthermore, we characterized ChiA as a bona fide chitinase that cleaves chitin but not chitosan and peptidoglycan, thus disproving its previously suggested role as a bifunctional endochitinase/ lysozyme.

\section{Results and Discussion}

Growth and Metabolite Accumulation Analyses of

E. coli Wild Type, chbG and chbF Mutants

To revisit the functions of ChbG and ChbF encoded by the $c h b$ operon, we first tested mutants deficient in either masses corresponding to GlcN-P $(\mathrm{M}+\mathrm{H})^{+} \mathrm{m} / \mathrm{z}=260.0362$ (light blue) and GlcNAc $(\mathrm{M}+\mathrm{H})^{+} \mathrm{m} / \mathrm{z}=222.0828$ (blue) appeared. The monoacetyl-chitobiose $(\mathrm{M}+\mathrm{H})^{+} \mathrm{m} / \mathrm{z}=383.1421$ (orange) instead remained intact. c Upon treatment with the exo-glucosaminidase NagZ (+NagZ), no GlcNAc was released from either of the accumulation products, indicating that GlcNAc is located at the reducing ends. Shown are the base peak chromatogram (BPC) mass range $(\mathrm{M}+\mathrm{H})^{+} \mathrm{m} / \mathrm{z}=120-800$ (gray) and the extracted ion chromatograms (EIC) based on the exact masses of displayed compounds (Table 1).

$c h b G$ or $c h b F$ for growth on diacetyl-chitobiose and chitobiose as the sole carbon source in M9 minimal medium, compared to the parental E. coli strain (wild type; wt). E. coli wt grew overnight to a final $\mathrm{OD}_{600}$ of 4.2 in $\mathrm{M} 9$ medium supplemented with $2 \mathrm{mM}$ diacetyl-chitobiose, while it failed to grow with chitobiose (data not shown). It has previously been described that $E$. coli wt grows with diacetyl-chitobiose but poorly with chitobiose as the only carbon source, presumably due to the lack of induction of the chb operon [Verma and Mahadevan, 2012]. As expected, the $\Delta c h b G:: k a n$ and $\Delta c h b F:: k a n$ strains were unable to grow on either diacetyl-chitobiose and chitobiose (data not shown). To identify accumulation products in $\Delta c h b G:: k a n$ and $\Delta c h b F:: k a n$, we grew the mutants overnight in M9 minimal medium with a mixture of amino acids (casamino acids; CAA) that was supplemented with $2 \mathrm{mM}$ of either diacetyl-chitobiose or triacetyl-chitotriose, isolated the cytosolic fractions, and analyzed them via high-performance liquid chromatography-mass spectrometry (HPLC-MS). In the cytosolic fraction of $\triangle c h b F:: k a n$ cells, we detected large amounts of compounds with masses corresponding to monoacetyl-chitobiose- $\mathrm{P}(\mathrm{M}+\mathrm{H})^{+}=463.1324 \mathrm{~m} / \mathrm{z}$, which appears as a double peak due to separation of the $\alpha$ - and $\beta$-anomeric 


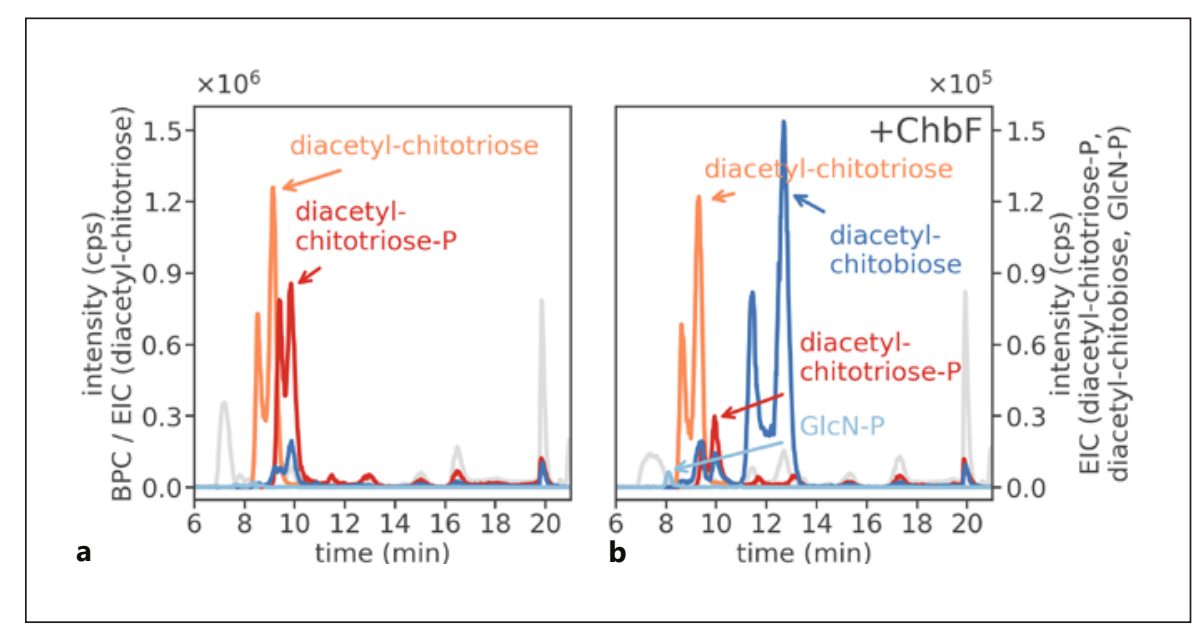

Fig. 2. Accumulation of diacetyl-chitotriose-P and diacetyl-chitotriose in $\triangle c h b F:: k a n$ cells and characterization of the ChbF products. The cytosolic fraction of E. coli $\Delta c h b F:$ kan cells, grown in M9 CAA minimal medium supplemented with triacetyl-chitotriose, was extracted and analyzed by LC-MS. a Accumulation products with masses $(\mathrm{m} / \mathrm{z})$ corresponding to diacetyl-chitotriose- $\mathrm{P}(\mathrm{M}+\mathrm{H})^{+}$ $\mathrm{m} / \mathrm{z}=666.2133$ (red) and diacetyl-chitotriose $(\mathrm{M}+\mathrm{H})^{+} \mathrm{m} / \mathrm{z}=$ 586.2453 (orange) were detected. The latter appeared as the major accumulation product. b Upon treatment with $\mathrm{ChbF}(+\mathrm{ChbF})$, the diacetyl-chitotriose- $\mathrm{P}(\mathrm{M}+\mathrm{H})^{+} \mathrm{m} / \mathrm{z}=666.2122$ (red) mostly disappeared, while masses corresponding to diacetyl-chitobiose $(\mathrm{M}+\mathrm{H})^{+}$

forms, and to monoacetyl-chitobiose $(\mathrm{M}+\mathrm{H})^{+}=383.1660$ $\mathrm{m} / \mathrm{z}$ (Fig. 1a; a list of compounds with their corresponding monoisotopic masses can be found in Table 1). These compounds are likely the products of the activity of the monodeacetylase $\mathrm{ChbG}$, which accumulate due to the lack of ChbF. The additional appearance of a dephosphorylated product indicates that a phosphatase partially cleaves the accumulating monoacetyl-chitobiose-P. To test whether the dephosphorylation event is growth phase-dependent, cytosolic fractions were isolated also from exponential phase-cells $\left(\mathrm{OD}_{600}=1\right)$. However, no difference in the relative amount of the phosphorylated and non-phosphorylated accumulation products was found in comparison to the stationary phase cells, indicating the expression of the enigmatic phosphatase is not growth phase-dependent (data not shown). Similarly, when the $\Delta c h b F:: k a n$ strain was grown in CAA-M9 medium supplemented with $2 \mathrm{mM}$ triacetyl-chitotriose, HPLC-MS analysis of cytosolic fractions revealed the presence of compounds with masses corresponding to diacetyl-chitotriose- $\mathrm{P}(\mathrm{M}+\mathrm{H})^{+}=666.2117 \mathrm{~m} / \mathrm{z}$ (Fig. 2a) and diacetyl-chitotriose $(\mathrm{M}+\mathrm{H})^{+}=586.2454 \mathrm{~m} / \mathrm{z}$, which both appear as double peaks due to separation of the $\alpha$ and $\beta$-anomeric forms. These compounds are the prod- $\mathrm{m} / \mathrm{z}=425.1790$ (blue) and GlcN-P $(\mathrm{M}+\mathrm{H})^{+} \mathrm{m} / \mathrm{z}=260.0543$ (light blue; a very small peak) appeared. The diacetyl-chitotriose $(\mathrm{M}+\mathrm{H})^{+}$ $\mathrm{m} / \mathrm{z}=586.2445$ (orange) was not affected by ChbF. A compound appeared with a mass corresponding to diacetyl-chitobiose that elutes at the same retention time as the diacetyl-chitotriose-P (blue), and thus is not diacetyl-chitobiose but an unknown component with the same mass. Shown are the base peak chromatogram (BPC) mass range $(\mathrm{M}+\mathrm{H})^{+} \mathrm{m} / \mathrm{z}=180-1,300$ (gray) and the extracted ion chromatograms (EIC) based on the exact masses of displayed compounds (Table 1).

ucts of the activity of the monodeacetylase ChbG, which apparently deacetylates the trisaccharide-P and possibly also the non-phosphorylated trisaccharide. The appearance of large amounts of diacetyl-chitotriose indicates that the enigmatic phosphatase preferentially cleaves diacetyl-chitotriose-P as compared to monoacetyl-chitobiose-P (cf. Fig. 1a).

Conversely, in the $\Delta c h b G:: k a n$ cytosolic fractions, the expected accumulation products, diacetyl-chitobiose- $\mathrm{P}$ and triacetyl-chitotriose-P could not be detected (data not shown). One could speculate that these saccharides do not accumulate because they are metabolized by an unknown, ChbG-independent pathway. If diacetyl-chitobiose is metabolized via a different pathway and thus is used as a source of energy and carbon, growth on this saccharide would be expected. The $\Delta c h b G:: k a n$ mutant, however, failed to grow with diacetyl-chitobiose. It has previously been reported that ChbG is crucial for the generation of monoacetyl-chitobiose, which supposedly acts as the inducer of the chb operon [Verma and Mahadevan, 2012]. Thus, the more plausible explanation for the absence of an accumulation product is that no induction occurred and, consequently, the ChbBCA-transporter was not expressed and diacetyl-chitobiose was not taken up. 
Table 1. Structures and exact monoisotopic masses of studied metabolites: overview of chb metabolites and their exact neutral masses and masses with proton adducts in positive ionization mode

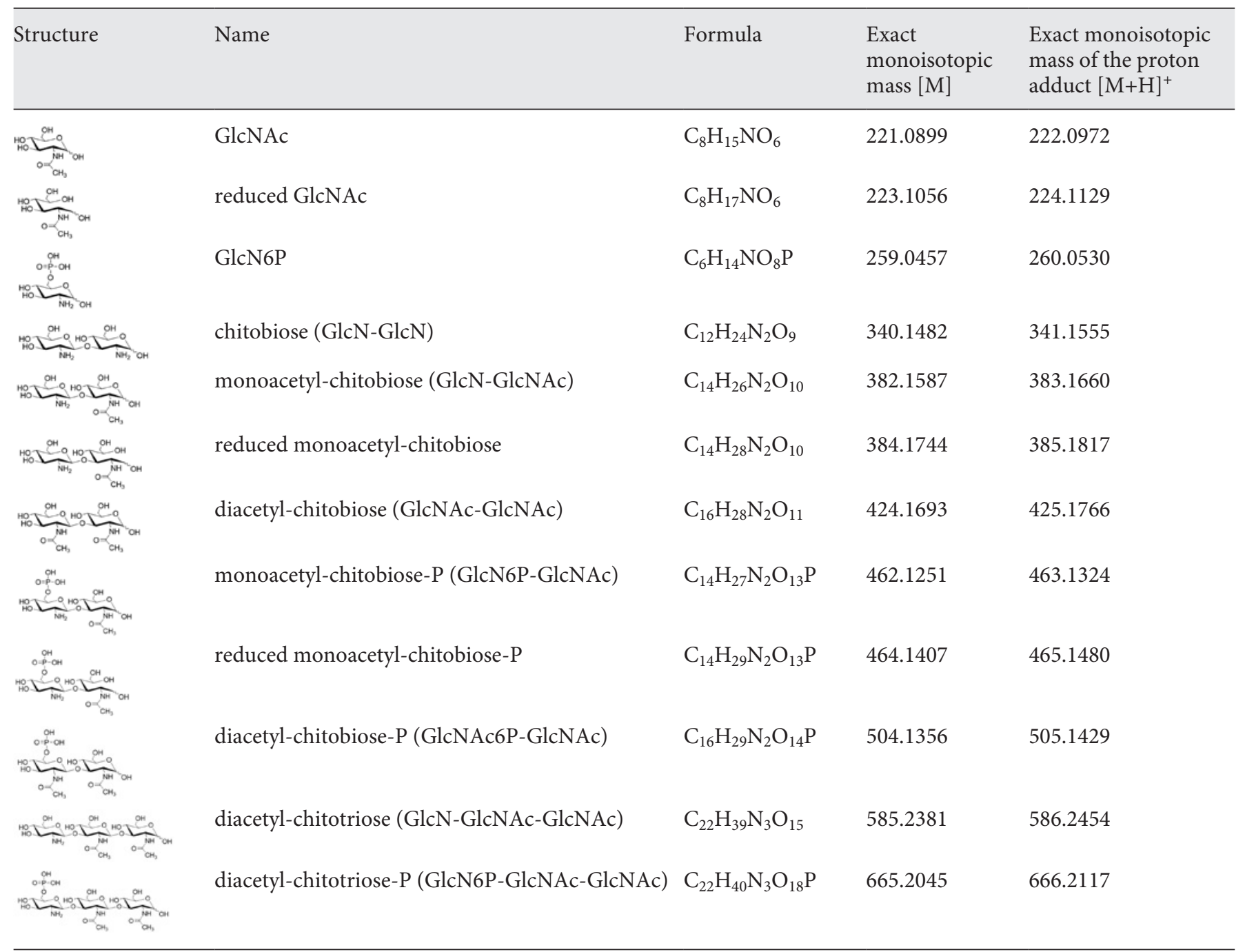

Deacetylation at the Non-Reducing End by ChbG Is Crucial for ChbF Function

To further characterize the accumulation products of the $\Delta c h b F:: k a n$ strain, the gene encoding the 6-phospho$\beta$-glycosidase ChbF (CelF) was cloned and recombinantly expressed. Recombinant ChbF completely degraded the phosphorylated accumulation product monoacetylchitobiose-P and yielded products with masses corresponding to $\mathrm{GlcN}-\mathrm{P}(\mathrm{M}+\mathrm{H})^{+} \mathrm{m} / \mathrm{z}=260.0530$ and GlcNAc $(\mathrm{M}+\mathrm{H})^{+} \mathrm{m} / \mathrm{z}=222.0972$, but was not able to cleave the non-phosphorylated accumulation product (Fig. 1b). It has previously been shown that diacetyl-chitobiose is phosphorylated at the non-reducing end during its transport into the cell by the ChbBCA-PTS system [Keyhani et al., 2000a]. On this basis, the cleavage of the accumulation product of $\triangle c h b F:: k a n$ into GlcN-P and GlcNAc by ChbF suggests that $\mathrm{ChbG}$ deacetylates diacetyl-chitobiose-P at the non-reducing end, yielding GlcN6P-GlcNAc. To unequivocally exclude that the dephosphorylated accumulation product of $\Delta c h b F:: k a n$ is GlcNAc-GlcN, we treated it with Bacillus subtilis exo- $N$-acetylglucosaminidase NagZ. This enzyme plays a role in the recycling of peptidoglycan and specifically cleaves off GlcNAc residues from the non-reducing end [Litzinger et al., 2010a, b]. While GlcNAc is released from diacetyl-chitobiose with NagZ (data not shown), no product is observed during digestion of the accumulation products of $\triangle c h b F:: k a n$, indicating the absence of GlcNAc entities at the non-reduc- 


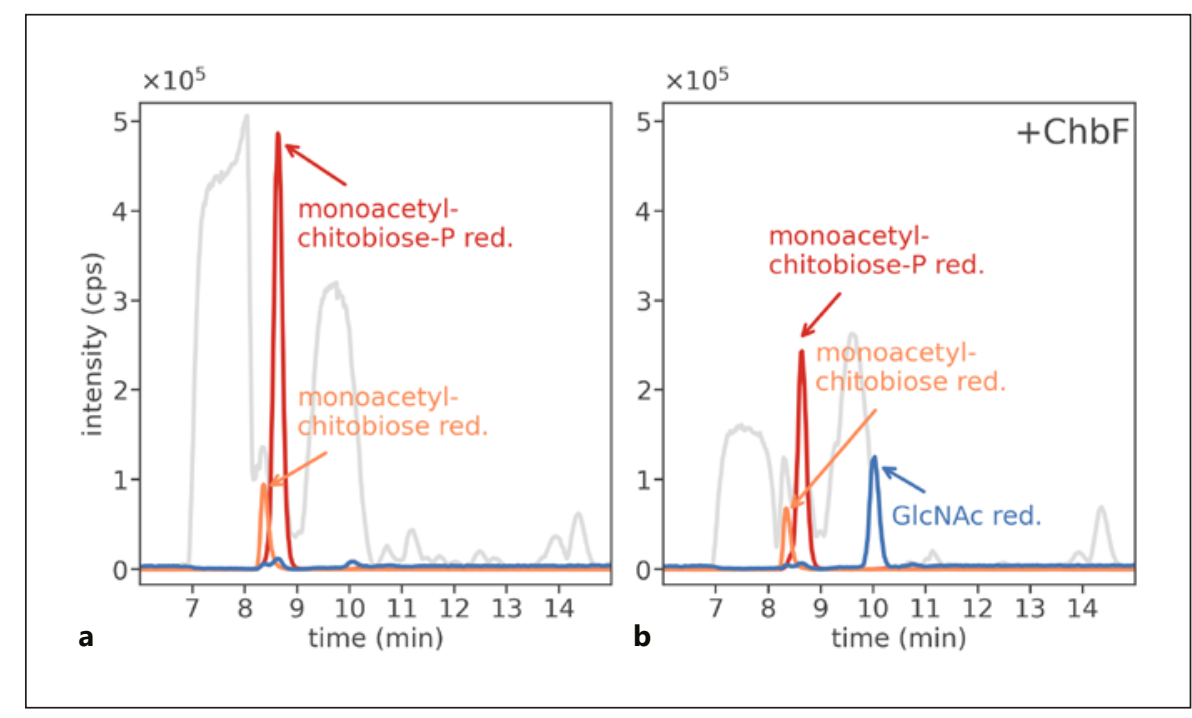

Fig. 3. Identification of the $\Delta c h b F:: k a n$ accumulation product as GlcNP-GlcNAc by reduction and LC-MS analysis of the reduced ChbF products. The cytosolic fraction of E. coli $\Delta$ chbF::kan cells containing monoacetyl-chitobiose-P and monoacetyl-chitobiose (see Fig. 1) was reduced with sodium-borohydride, incubated with ChbF and analyzed by LC-MS. a Both, the reduced forms of monoacetyl-chitobiose- $\mathrm{P}(\mathrm{M}+\mathrm{H})^{+} \mathrm{m} / \mathrm{z}=465.1157$ (red) and monoacetyl-chitobiose $(\mathrm{M}+\mathrm{H})^{+} \mathrm{m} / \mathrm{z}=385.1552$ (orange) were detected. They appear as single peaks, since $\alpha$ and $\beta$ anomers yield the same product after reduction. $\mathbf{b}$ Upon treatment with $\mathrm{ChbF}$ the peak of

ing terminus (Fig. 1c). Similarly, it was shown that ChbF is able to cleave diacetyl-chitotriose- $\mathrm{P}$, which accumulates in E. coli $\Delta$ chbF::kan cells grown in the presence of triacetyl-chitotriose, yielding diacetyl-chitobiose and GlcN-P (Fig. 2b). This indicates that triacetyl-chitotriose is also phosphorylated and deacetylated at the non-reducing end by the ChbBCA-PTS and ChbG, respectively, yielding GlcN6P-GlcNAc-GlcNAc, from which GlcN6P at the non-reducing end is cleaved-off by $\mathrm{ChbF}$.

A method to identify the sugar at the reducing end beyond doubt is the reduction with sodium borohydride followed by enzymatic cleavage and/or fragmentation by tandem MS (MS/MS). In these conditions, the reduction of the accumulation products of $\triangle c h b F:: k a n$ was complete and the two reduced products could be detected (Fig. 3a). When ChbF was added, the peak of the phosphorylated, reduced substrate became smaller and a distinct peak emerged, with a mass that corresponds to reduced GlcNAc $(\mathrm{M}+\mathrm{H})^{+} \mathrm{m} / \mathrm{z}=224.1129$ (Fig. 3b). A mass for GlcN-P, which would also be expected, was not detected. GlcN-P does not ionize very well in MS and its ionization could have been worsened in the samples that were reduced with sodium borohydride due to high salt reduced monoacetyl-chitobiose- $\mathrm{P}(\mathrm{M}+\mathrm{H})^{+} \mathrm{m} / \mathrm{z}=465.1163$ (red) decreased significantly, but not the reduced monoacetyl-chitobiose $(\mathrm{M}+\mathrm{H})^{+} \mathrm{m} / \mathrm{z}=385.1555$ (orange), and reduced GlcNAc $(\mathrm{M}+\mathrm{H})^{+} \mathrm{m} / \mathrm{z}=224.0974$ (blue) appeared. Notably, the second breakdown product, glucosamine-phosphate (GlcN-P) could not be detected. Shown are the base peak chromatogram (BPC) mass range $(\mathrm{M}+\mathrm{H})^{+} \mathrm{m} / \mathrm{z}=120-800$ (gray) and the extracted ion chromatograms (EIC) based on the exact masses of displayed compounds (Table 1).

concentrations. These conditions are also suboptimal for ChbF and, accordingly, the substrate was not completely digested by ChbF. Nevertheless, the detection of reduced GlcNAc $\left([\mathrm{M}+\mathrm{H}]^{+} \mathrm{m} / \mathrm{z}=224.1129\right)$ in significant abundance $(83 \%$ relative to the reduced monoacetyl-chitobiose-P, which was set to $100 \%$ ) indicates that there had been GlcNAc at the reducing end of the ChbF substrate (Fig. 4). Both compounds could also be detected in anhydrous forms with lower intensities. The mass difference between reduced monoacetyl-chitobiose- $\mathrm{P}$ and reduced GlcNAc corresponds exactly to uncharged GlcN-P $-\mathrm{H}_{2} \mathrm{O}$ $(\mathrm{M})=241.0351 \mathrm{Da}$. Furthermore, the mass of GlcN-P $-\mathrm{H}_{2} \mathrm{O}$ itself could be detected, although in very low abundance of $<0.5 \%$. This indicates once more that GlcNAc ionizes readily in MS positive ion mode compared to GlcN-P.

In summary, our results indicate that ChbG deacetylates its substrate diacetyl-chitobiose-P at the non-reducing end. This finding fits well into the previous reports that $E$. coli is able to utilize the $\beta$-glucoside sugars cellobiose, arbutin and salicin by expressing the chb (formerly designated cel) operon [Parker and Hall, 1990]. In each of these sugars, the non-reducing end 


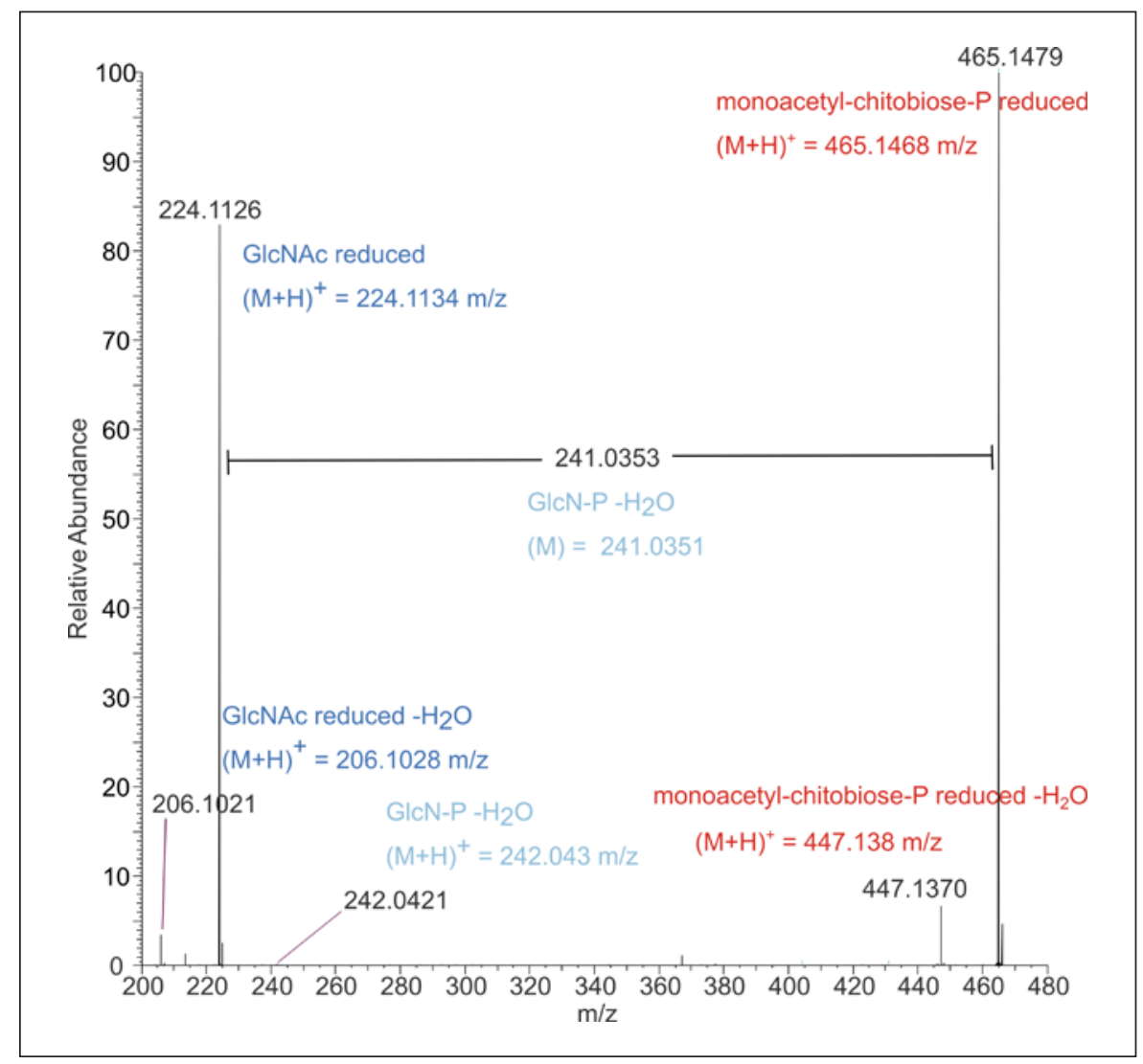

Fig. 4. MS-MS fragmentation analysis of the reduced $\Delta c h b F:: k a n$ accumulation product monoacetyl-chitobiose-P. In order to identify the position of deacetylation in the monoacetyl-chitobiose-P, the $\Delta c h b F:: k a n$ accumulation product was reduced with sodium borohydride (cf. Fig. 3) and analyzed by MS-MS. Reduced monoacetyl-chitobiose- $\mathrm{P}(\mathrm{M}+\mathrm{H})^{+}=465.1480 \mathrm{~m} / \mathrm{z}$ (red) was set to $100 \%$ relative abundance. MS-MS fragmentation results in the formation of reduced GlcNAc $(\mathrm{M}+\mathrm{H})^{+} \mathrm{m} / \mathrm{z}=224.1129$ (blue), appearing

consists of glucose, while the residue at the reducing end is variable. These $\beta$-glucosides, after phosphorylation, were shown to be substrates for ChbF (previously named CelF) [Thompson et al., 1999]. Our data also fit well into the proposed enzymatic mechanism of GH4 members, which posits that the decisive reaction mechanism takes place at the carbohydrate at the non-reducing end. Indeed, oxidation/reduction of the hydroxyl group at C3 of the sugar at non-reducing end of a disaccharide to a carbonyl renders the $\mathrm{C} 2$ proton more acidic and thereby facilitates the elimination of the glycosidic oxygen, that is, the cleavage of the glycosidic bond [Rajan et al., 2004; Yip et al., 2004, 2007]. In the second step of the proposed mechanism, a proton gets released from the non-reducing end sugar's $\mathrm{C} 2$ hydrogen to a with a relative abundance of $83 \%$, which results by loss of uncharged GlcN-P $-\mathrm{H}_{2} \mathrm{O}(\mathrm{M})=241.0351 \mathrm{Da}$ (light blue). Detected were the mass of GlcN-P $-\mathrm{H}_{2} \mathrm{O}$ (light blue) $(\mathrm{M}+\mathrm{H})^{+} \mathrm{m} / \mathrm{z}=242.0424$ in very low abundance $(<0.5 \%)$ and of anhydrous forms of reduced monoacetyl-chitobiose- $\mathrm{P}(\mathrm{M}+\mathrm{H})^{+} \mathrm{m} / \mathrm{z}=447.1374$ (red) and reduced GlcNAc $(\mathrm{M}+\mathrm{H})^{+} \mathrm{m} / \mathrm{z}=206.1023$ in low relative abundances of 7 and $4.5 \%$, respectively.

catalytic base. This process is favored by a positive dipole on the $\mathrm{C} 2$, which is more pronounced with an amino instead of a $\mathrm{N}$-acetyl functional group. The binding of $\mathrm{Mn}^{2+}$ to $\mathrm{C} 2$ and C3 functional groups would also be enhanced with the more negative dipole on the amino group. A well-studied member of GH4 is the 6-phosphoa-glucosidase GlvA from B. subtilis [Yip et al., 2007]. The crystal structure of GlvA bound to glucose 6-phosphate (Glc6P) revealed that at C2 there is relatively little space in the binding pocket and steric hindrance could occur with larger side chains. An amino acid sequence alignment among ChbF, GlvA, and other described GH4 enzymes revealed high similarities especially at the site next to the $\mathrm{C} 2$ functional group, where a cysteine residue is conserved across all queried enzymes. To 


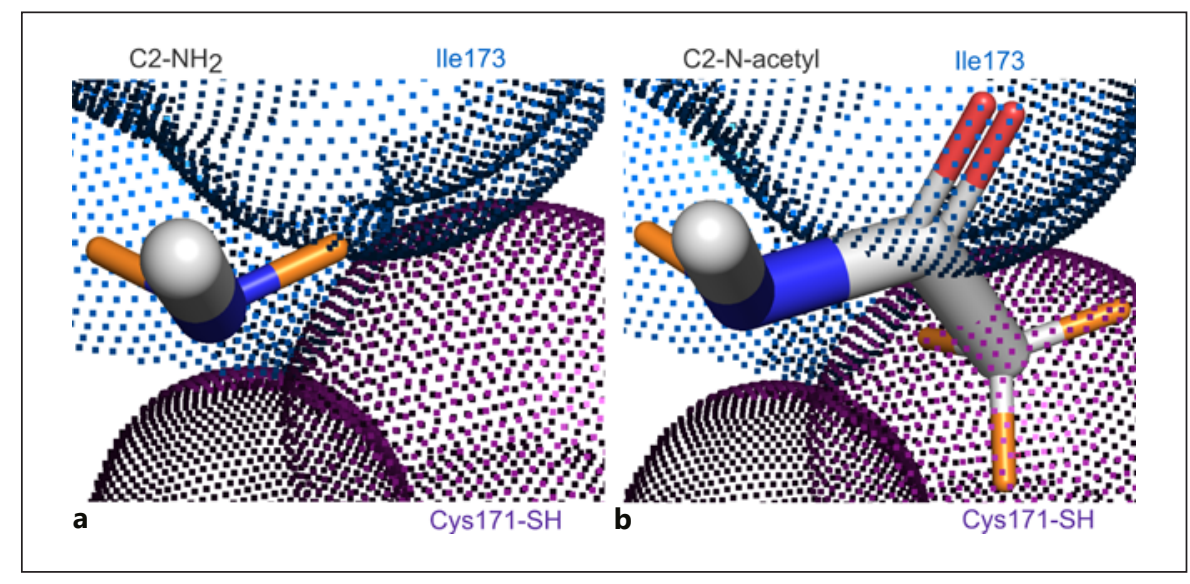

Fig. 5. Structural model of the binding pocket of ChbF in complex with substrates. Based on the crystal structure of the 6-phospho-aglucosidase GlvA from B. subtilis in complex with its substrate glucose-6P (pdb code: 1U8X), the binding pocket of ChbF was modeled using Pymol, identifying steric constraints that discriminate binding of an $\mathrm{N}$-acetyl group, but allow binding of an amino or hydroxyl group. a, b Shown is the $\mathrm{C} 2$ with the functional group shown as sticks (carbon $=$ white, nitrogen $=$ blue, oxygen $=$ red, hydrogen $=$ orange) and the amino acid residues isoleucine (blue) and cysteine (purple) in direct contact with this functional group at $\mathrm{C} 2$, shown as dotted spheres. Alignments between the amino

further clarify the difference between amino and $\mathrm{N}$-acetyl groups at $\mathrm{C} 2$ for $\mathrm{ChbF}$ we modeled its structure using the GlvA-Glc6P cocrystal structure (pdb code: $1 \mathrm{U} 8 \mathrm{X}$ ) and exchanging the $\mathrm{C} 2$ hydroxyl group of the ligand with an amino or $N$-acetyl group. Two amino acids are in proximity of the functional group, the conserved cysteine and methionine in GlvA, the latter replaced with the isoleucine of ChbF. This modeling suggests that an amino group fits well between the residues (Fig. 5a), whereas the $N$-acetyl group on the other hand does not, as the oxygen collides with the isoleucine and the methyl group collides with the sulfur of cysteine (Fig. 5b).

To test in vivo the enzymatic activity of ChbF on diacetyl-chitobiose-P, E. coli $\Delta$ chbG::kan was transformed with a plasmid that allows IPTG-inducible expression of the ChbBCA-PTS. Since under these conditions, due to the missing ChbR coactivator, the natural induction of the chb operon does not occur, we expected the accumulation of diacetyl-chitobiose-P, when grown overnight in M9 CAA minimal medium supplemented with diacetylchitobiose. Accordingly, diacetyl-chitobiose-P $(\mathrm{M}+\mathrm{H})^{+}$ $\mathrm{m} / \mathrm{z}=505.1429$ accumulated in the cytosolic fraction (Fig. 6a). Intriguingly, $\mathrm{ChbF}$ is not able to digest this compound (Fig. 6b), which confirms the inability of ChbF to acid sequences of ChbF, GlvA, and other representatives of the GH4 family enzymes show a high degree of similarity and especially a conserved cysteine residue (ChbF Cys172, GlvA Cys171) close to the $\mathrm{C} 2$ functional group. Also close are Ile174 of ChbF and Met173 of GlvA. In this structure model, Met173 GlvA was exchanged with Ile to resemble the situation of ChbF. a The $\mathrm{C} 2$ functional group has been altered to an amino group like in $\mathrm{GlcN}$, which fits well between the Ile and Cys residues. $\mathbf{b}$ The $\mathrm{C} 2$-functional group has been altered to $\mathrm{N}$-acetyl group of GlcNAc, which, however, does not fit since the oxygen of the acetyl group collides with the Ile and the methyl group collides with the sulfur of Cys.

take substrates with an $\mathrm{N}$-acetyl group at the non-reducing end. Notably, as seen throughout in the accumulation studies, also here phosphorylated and non-phosphorylated saccharide represented the major accumulation product. This further suggests the action of a phosphatase that partially dephosphorylates the accumulation product GlcNAc6P-GlcNAc.

\section{ChiA Cleaves Chitin and Partially Acetylated}

Chitosan but Not Fully Deacetylated Chitosan

E. coli ChiA was previously characterized as an endochitinase, since it cleaved the artificial chitinase substrate 4-methylumbelliferyl (4-MU)-(GlcNAc) $)_{3}$ but not 4-MUGlcNAc [Francetic et al., 2000a]. Furthermore, in the latter report, ChiA was described as a bifunctional chitinase/ lysozyme, on the basis of its ability to cleave ethyleneglycol chitin (EGC), which is a common lysozyme substrate [Wang and Chang, 1997]. We aimed to re-evaluate the enzyme's specificity using natural substrates, chitin (-oligomers), chitosan (-oligomers), and peptidoglycan, and thus expressed and purified ChiA as a polyhistidinetagged recombinant protein. Hydrolysis of 4-MU$(\mathrm{GlcNAc})_{3}$ (Sigma-Aldrich) was confirmed with the recombinant protein (data not shown). A preferred substrate of chitinases is colloidal chitin, which was prepared 


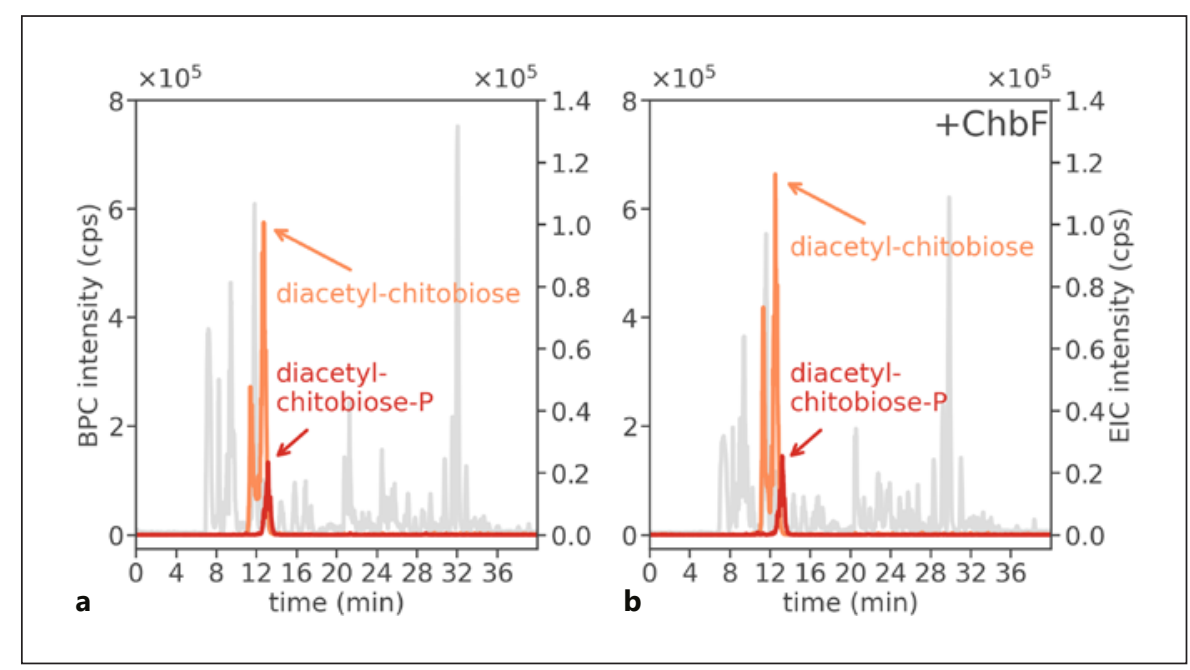

Fig. 6. ChbF is unable to cleave diacetyl-chitobiose-P. a Diacetylchitobiose $(\mathrm{M}+\mathrm{H})^{+} \mathrm{m} / \mathrm{z}=425.1776$ (orange) and diacetyl-chitobiose- $\mathrm{P}(\mathrm{M}+\mathrm{H})^{+} \mathrm{m} / \mathrm{z}=505.1445$ (red) accumulate in cytosolic extracts of strain E. coli $\triangle c h b G:: k a n$ carrying pQE32-chbBCA and grown in M9 CAA medium in the presence of IPTG and diacetylchitobiose. $\mathbf{b}$ Incubation of the extract in $\mathbf{a}$ with recombinant $\mathrm{ChbF}$ does not result in a decrease in the intensity of the mass peak of diacetyl-chitobiose $(\mathrm{M}+\mathrm{H})^{+} \mathrm{m} / \mathrm{z}=425.1780$ (orange) and diacetylchitobiose- $\mathrm{P}(\mathrm{M}+\mathrm{H})^{+} \mathrm{m} / \mathrm{z}=505.1445$ (red). Shown are the base peak chromatogram (BPC) mass range $(\mathrm{M}+\mathrm{H})^{+} \mathrm{m} / \mathrm{z}=120-800$ (gray) and the extracted ion chromatograms (EIC) based on the exact masses of displayed compounds (Table 1). by the acetylation of low molecular weight chitosan $(<3$ $\mathrm{kDa}$ in size; obtained from Carbosynth). ChiA was able to cleave colloidal chitin yielding diacetyl-chitotriose and triacetyl-chitotriose (Fig. 7b), whereas an endo-chitinase from Streptomyces griseus, in contrast, almost exclusively yielded diacetyl-chitobiose (Fig. 7c). That ChiA releases dimers and trimers in approximately equal amounts could be due to the fact that the enzyme features five chitin-binding type-3 domains which mediate binding to the glycan chains. To understand whether ChiA requires acetylated sugars, we incubated it with chitosan oligomers up to $3 \mathrm{kDa}$ in size. The oligomer mix contained large amounts of chitotriose and chitotetraose and smaller amounts of chitobiose (Fig. 8a). Incubation with ChiA did not result in the release of small chitosan-oligosaccharides, but released significant amounts of diacetyl-chitotriose (Fig. 8b). As the diacetyl-chitotriose product of ChiA could not be further cleaved by the exo- $N$-acetylglucosaminidase NagZ from $B$. subtilis, we suggest the diacetyl-chitotriose product of ChiA is composed of $\mathrm{GlcN}-(\mathrm{GlcNAc})_{2}$. These data indicate that ChiA is able to cleave particular bonds within polymeric, acetylated chitosan releasing products that have GlcNAc at the reducing end. As a positive control, the chitosanase Csn from B. subtilis was cloned, expressed, and purified and the digestion was performed according to the conditions described [Rivas et al., 2000]. After incubation of the chito- san oligomers with Csn an increased amount of chitobiose could be measured, while chitotetraose was completely digested (Fig. 8c).

\section{ChiA Has No Lysozyme Activity}

To investigate the lysozyme activity of ChiA, peptidoglycan was purified from E. coli and B. subtilis. Unlike the positive controls, Atl (Glc), the glucosaminidase domain of the bifunctional $\mathrm{N}$-acetylmuramoyl-L-alanine amidase/endo- $\beta-N$-acetylglucosaminidase Atl from Staphylococcus aureus [Oshida et al., 1995], and mutanolysin, the endo- $N$-acetymuramidase from Streptomyces sp. [Rau et al., 2001], ChiA did not release products from the peptidoglycans of either source. From E. coli peptidoglycan, Atl (Glc) released MurNAc-GlcNAc in smaller and MurNAc-GlcNAc-tetrapeptide ([MurNAc-GlcNAc]$4 \mathrm{P}$ ) in larger quantities (Fig. 9c). From B. subtilis peptidoglycan, Atl released disaccharides with and without peptides (online suppl. Fig. S1C; for all online suppl. material, see www.karger.com/doi/10.1159/000515178). To test if ChiA possibly only cleaves denuded peptidoglycan, that is, poly-GlcNAc-MurNAc chains devoid of peptides, the $\mathrm{N}$-acetylmuramoyl-L-alanine amidase CwlC from $B$. subtilis [Shida et al., 2000] was used to remove the peptide stems from the peptidoglycan as reported elsewhere [Müller et al., 2021]. CwlC quantitatively released tripeptides (3P) and tetrapeptides (4P) as well as crosslinked 


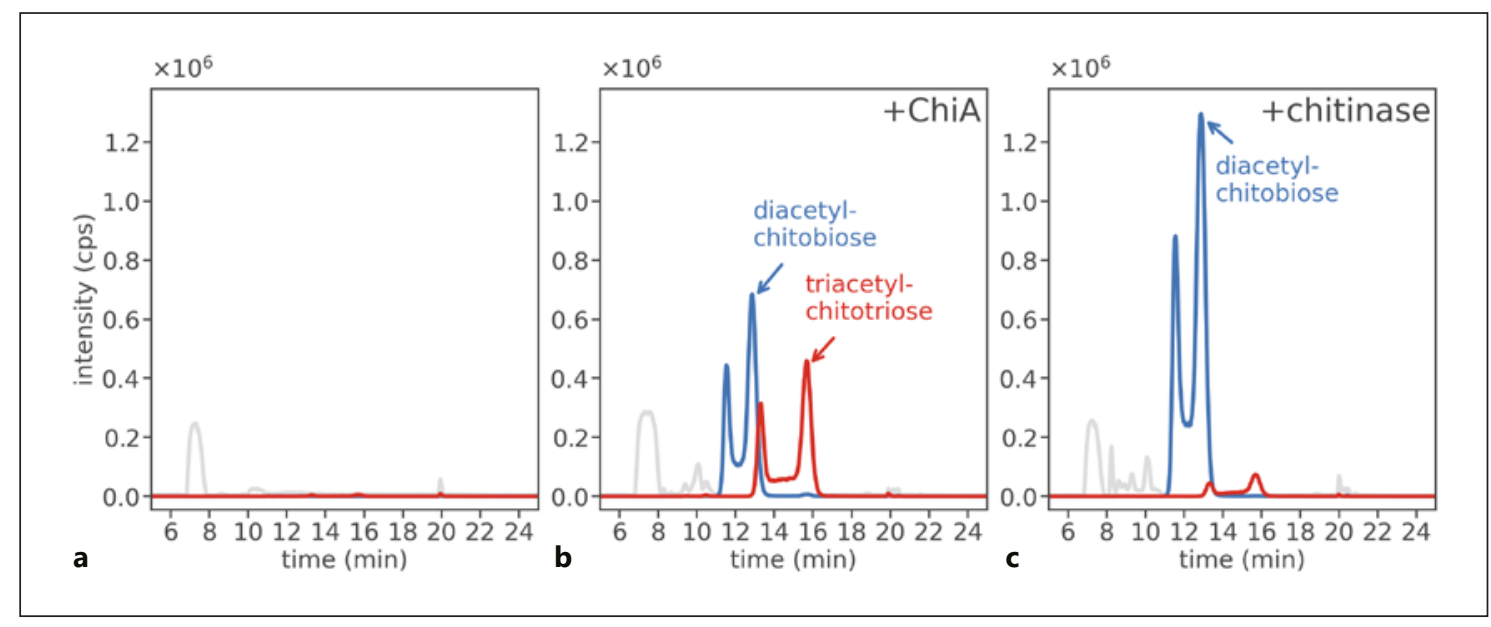

Fig. 7. ChiA cleaves colloidal chitin releasing diacetyl-chitobiose and triacetyl-chitotriose. Colloidal chitin was incubated with ChiA and a chitinase from S. griseus and the reaction products were analyzed by LC-MS. a Untreated colloidal chitin does not contain soluble chitooligosaccharides, detectable by MS. b After a 2-hour digestion of colloidal chitin with ChiA diacetyl-chitobiose $(\mathrm{M}+\mathrm{H})^{+}$ $\mathrm{m} / \mathrm{z}=425.1769$ (blue) and triacetyl-chitotriose $(\mathrm{M}+\mathrm{H})^{+} \mathrm{m} / \mathrm{z}=$
628.2573 (red) are formed, but larger oligomers are not visible. c A chitinase from $S$. griseus (the positive control) almost exclusively released diacetyl-chitobiose $(\mathrm{M}+\mathrm{H})^{+} \mathrm{m} / \mathrm{z}=425.1765$ (blue) from colloidal chitin. Shown are the base peak chromatogram (BPC) mass range $(\mathrm{M}+\mathrm{H})^{+} \mathrm{m} / \mathrm{z}=199-1,300$ (gray) and the extracted ion chromatograms (EIC) based on the exact masses of displayed compounds (online suppl. Table S2).

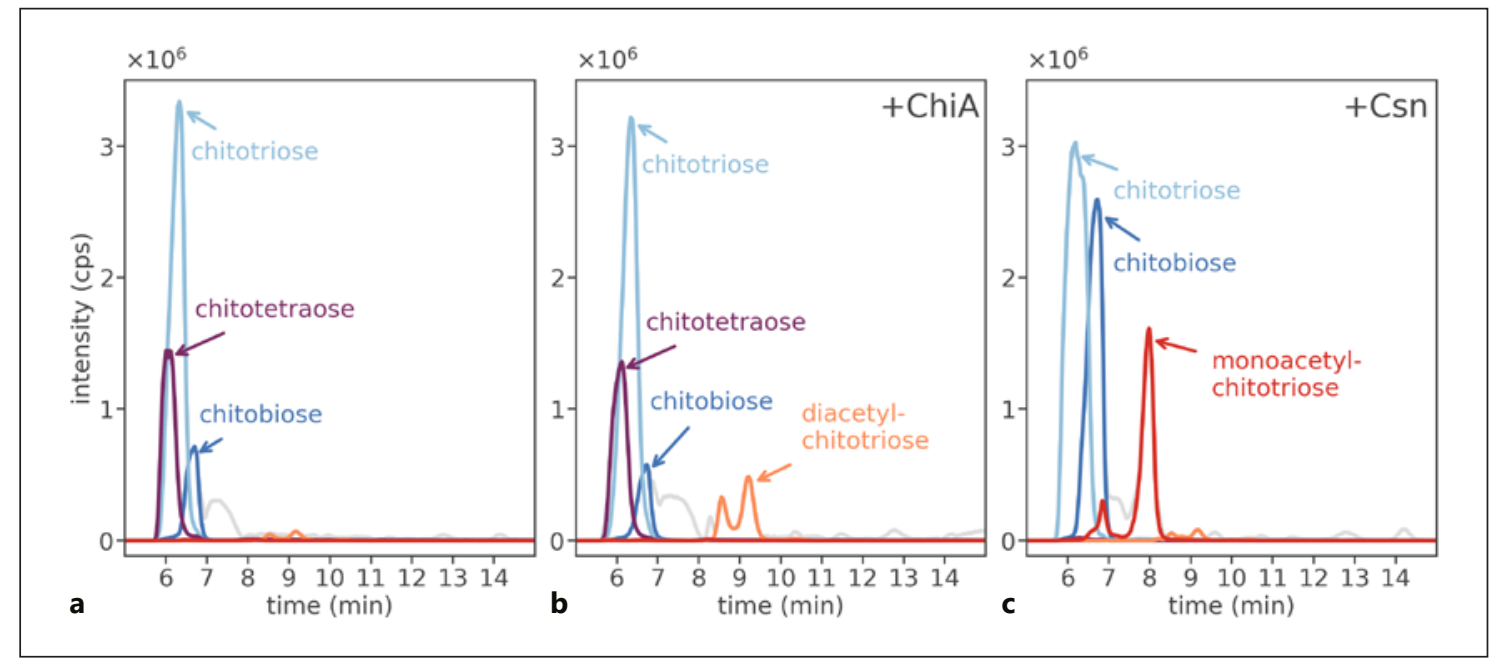

Fig. 8. ChiA cleaves partially acetylated chitosan yielding diacetylchitotriose. Chitosan $(<3 \mathrm{kDa})$ was incubated with ChiA and a chitosanase from $B$. subtilis and the reaction products were analyzed by LC-MS. a Untreated chitosan (control) already contains small amounts of chitobiose $(\mathrm{M}+\mathrm{H})^{+} \mathrm{m} / \mathrm{z}=341.1576$ (blue), chitotriose $(\mathrm{M}+\mathrm{H})^{+} \mathrm{m} / \mathrm{z}=502.2226$ (light blue), and chitotetraose $(\mathrm{M}+\mathrm{H})^{+}$ $\mathrm{m} / \mathrm{z}=663.2943$ (purple). $\mathbf{b}$ Upon digestion of partially acetylated chitosan with ChiA (+ChiA), the amounts of deacetylated products were not increased, but the release of diacetyl-chitotriose
$(\mathrm{M}+\mathrm{H})^{+} \mathrm{m} / \mathrm{z}=586.2508$ (GlcN-GlcNAc-GlcNAc; orange) was detected. c Upon digestion of partially acetylated chitosan with $B$. subtilis chitosanase Csn the amount of chitobiose $(\mathrm{M}+\mathrm{H})^{+} \mathrm{m} / \mathrm{z}=$ 341.1560 (blue) and also monoacetyl-chitotriose $(\mathrm{M}+\mathrm{H})^{+} \mathrm{m} / \mathrm{z}=$ 544.2386 (red) were significantly increased, while the chitotetraose was completely digested. Shown are the base peak chromatogram (BPC) mass range $(\mathrm{M}+\mathrm{H})^{+} \mathrm{m} / \mathrm{z}=199-1,300$ (gray) and the extracted ion chromatograms (EIC) based on the exact masses of displayed compounds (online suppl. Table S2). 
Fig. 9. ChiA does not cleave peptidoglycan. Intact peptidoglycan, derived from $E$. coli (left panels), and peptide-denuded peptidoglycan (right panels), that is, treated with amidase CwlC to remove the peptide stems, were incubated with ChiA or the endo-glucosaminidase Atl (Glc) of S. aureus. The formation of reaction products was analyzed by LC-MS. a Uncleaved E. coli peptidoglycan as a control. b Incubation of $E$. coli peptidoglycan with ChiA (+ChiA) does not release any soluble products. c Incubation of E. coli peptidoglycan with Atl (Glc) $(+$ Atl $(\mathrm{Glc})$ ), releases large amounts of (MurNAc-GlcNAc)-tetra-peptide $(\mathrm{M}+\mathrm{H})^{+}$ $\mathrm{m} / \mathrm{z}=940.3922$ ((MurNAc-GlcNAc)-4P; blue) and to a lesser extent also MurNAcGlcNAc $(\mathrm{M}+\mathrm{H})^{+} \mathrm{m} / \mathrm{z}=497.1954$ (red). d CwlC $(+\mathrm{CwlC})$ removes tri- $(\mathrm{M}+\mathrm{H})^{+} \mathrm{m} / \mathrm{z}$ $=391.1650(3 \mathrm{P}$, light blue $)$, tetra $-(\mathrm{M}+\mathrm{H})^{+}$ $\mathrm{m} / \mathrm{z}=462.2006$ (4P, orange), tri-tetra $(\mathrm{M}+\mathrm{H})^{+} \mathrm{m} / \mathrm{z}=834.3448$ (3P-4P, yellow), and tetra-tetra peptides $(\mathrm{M}+\mathrm{H})^{+} \mathrm{m} / \mathrm{z}=$ 905.3808 (4P-4P, purple) from $E$. coli peptidoglycan. e From d, ChiA (+CwlC+ChiA) is unable to cleave denuded peptidoglycan. f From d, Atl (Glc) $(+\mathrm{CwlC}+\mathrm{Atl}(\mathrm{Glc}))$ is able to release large amounts of MurNAc$\operatorname{GlcNAc}(\mathrm{M}+\mathrm{H})^{+} \mathrm{m} / \mathrm{z}=497.1750$ (red). Intriguing, no MurNAc-GlcNAc-4P could be detected, indicating the complete removal of the stem peptides from the peptidoglycan by CwlC. Shown are the base peak chromatograms (BPC) mass range $(\mathrm{M}+\mathrm{H})^{+}$ $\mathrm{m} / \mathrm{z}=200-2,000$ (gray) and the extracted ion chromatograms (EIC) based on the exact masses of displayed compounds (online suppl. Table S2).
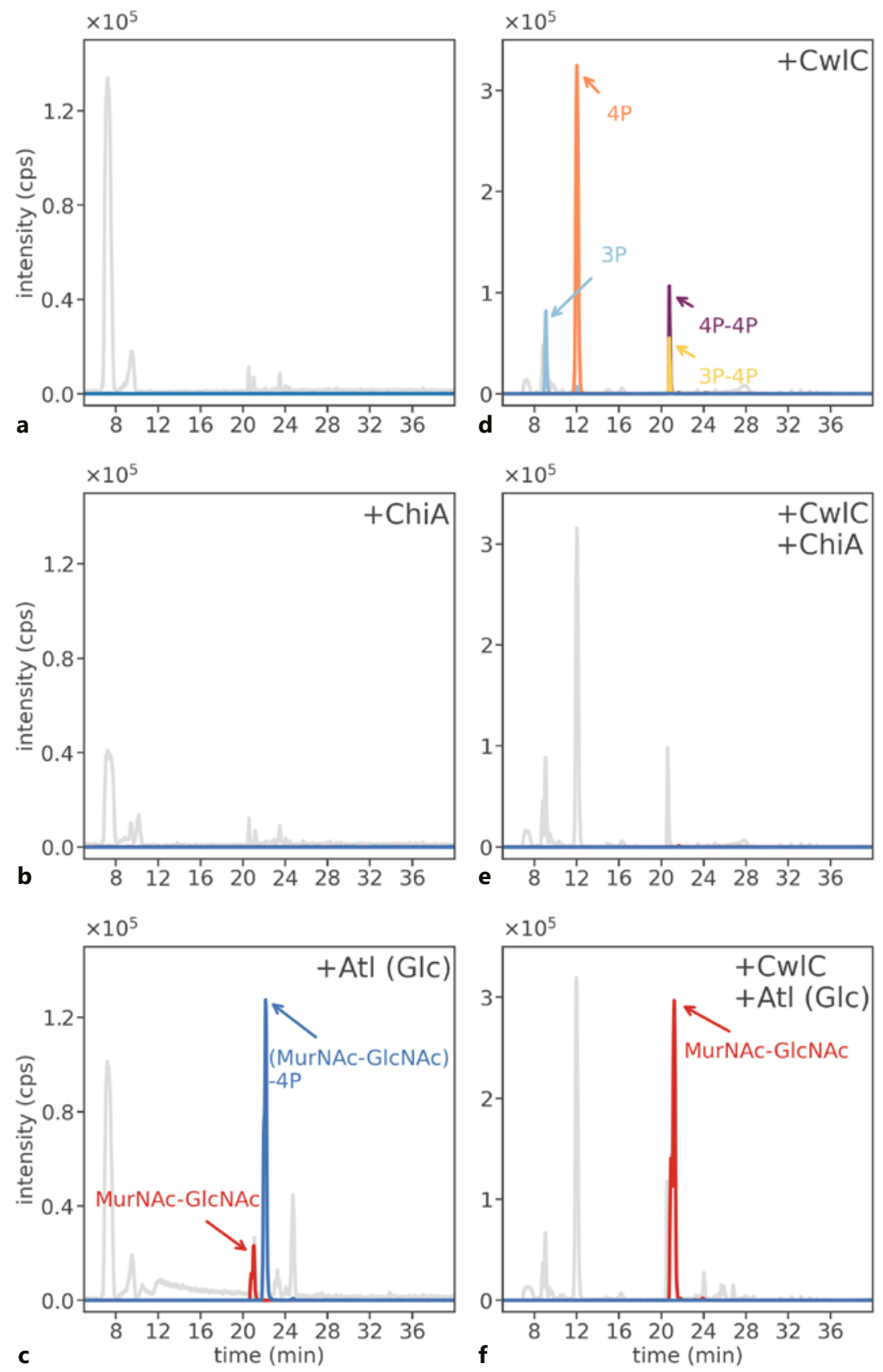

4P-4P and 3P-4P peptides (Fig. 9d) from E. coli peptidoglycan. From B. subtilis peptidoglycan it released 3P*, $3 \mathrm{P}^{*}-4 \mathrm{P}^{*}$, and $3 \mathrm{P}-4 \mathrm{P}^{*}$, in which the amino acid meso-diaminopimelic acid is amidated (assigned with ${ }^{*}$ ) (online suppl. Fig. S1E). However, ChiA was not able to release any products from denuded peptidoglycan chains (Fig. 9e; online suppl. Fig. S1F). Atl (Glc), on the other hand, re- leases significant amounts of MurNAc-GlcNAc as the only product in both cases. The fact that no disaccharide with peptides was released using Atl (Glc) indicates that CwlC has completely removed the peptides (Fig. 9f; online suppl. Fig. S1G). Mutanolysin, in contrast, cleaved native peptidoglycan, but was unable to degrade denuded peptidoglycan (online suppl. Fig. S1H). In summary, the 
Fig. 10. ChiA is generally not expressed in $E$. coli wt, but periplasmic expression facilitates growth on penta-acetyl-chitopentaose $\left(\mathrm{GlcNAc}_{5}\right.$ ). a E. coli wild-type (red), $\Delta c h i A:: k a n$ (blue), $\Delta c h b G:: k a n$ (light blue), $\Delta c h b F:: k a n$ (orange), and BL21 (DE3) pET28a-chiA (purple) strains were grown in minimal medium supplemented with $0.1 \%$ glucose $(+\mathrm{Glc})$ as the only carbon source. wt, $\Delta c h b G:: k a n, \Delta c h b F:: k a n$, and $\Delta c h i A:: k a n$ show a similar growth behavior reaching stationary phase after $7 \mathrm{~h}$. b In minimal medium supplemented with $0.1 \%$ glucose and $0.2 \%$ diacetyl-chitobiose $\left(+\mathrm{Glc}+(\mathrm{GlcNAc})_{2}\right)$, wt and $\triangle c h i A:: k a n$ showed biphasic growth, whereas $\Delta c h b G:: k a n$ and $\Delta c h b F:: k a n$ grew as in medium with sole glucose. c In minimal medium supplemented with $0.1 \%$ glucose and $0.2 \%$ penta-acetyl-chitopentaose $\left(+\mathrm{Glc}+(\mathrm{GlcNAc})_{5}\right)$, wt and $\Delta c h i A:: k a n$ grew as in medium with sole glucose, indicating that penta-acetyl-chitopentaose is not utilized. d In minimal medium supplemented with $0.1 \% \mathrm{CAA}$ and $0.2 \%$ penta-acetyl-chitopentaose (+CAA+(GlcNAc) $\left.)_{5}+\mathrm{IPTG}\right)$, again, E. coli wt is not able to utilize the penta-acetyl-chitopentaose; however, periplasmic expression of ChiA from a plasmid facilitated growth on penta-acetyl-chitopentaose, resulting in a biphasic growth and a significantly higher final $\mathrm{OD}_{600}$ of 0.3 .
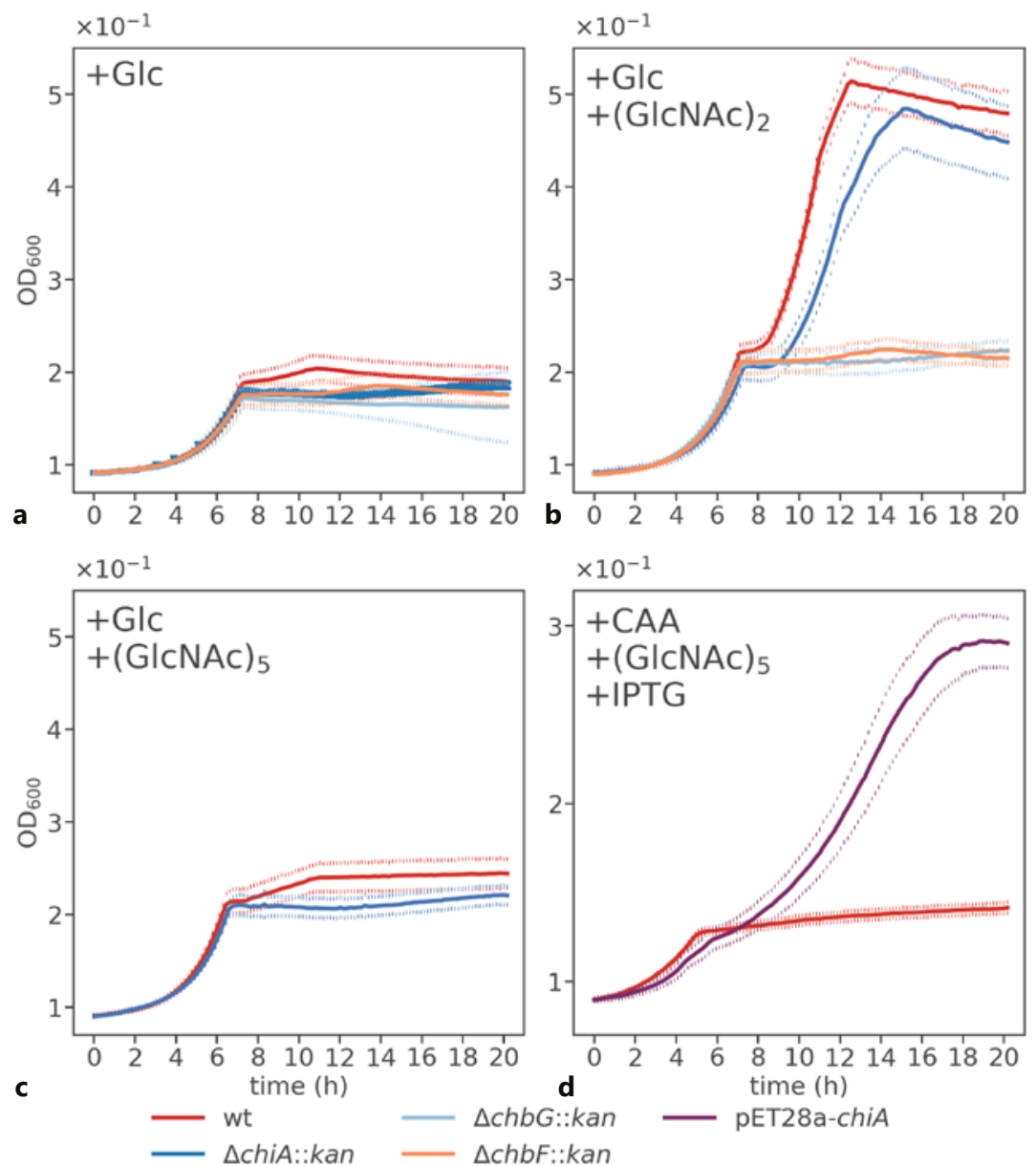

results show that ChiA can digest chitin but neither chitosan nor peptidoglycan polymers irrespectively of whether they are native or denuded.

\section{ChiA Expression Facilitates Growth on}

\section{Penta-Acetyl-Chitopentaose}

With the sole role of ChiA as chitinase confirmed in vitro, the growth of E. coli wt and $\Delta c h i A:: k a n$ in M9 glucose minimal medium was compared in the presence of diacetyl-chitobiose or penta-acetyl-chitopentaose. As expected both strains were able to grow on glucose and diacetyl-chitobiose exhibiting a biphasic growth pattern due to carbon catabolite repression (Fig. 10b). With penta-acetyl-chitopentaose no biphasic growth was observed, as only glucose was utilized (Fig. 10c). However, the wt strain was expected to utilize pentaacetyl-chitopentaose if ChiP and ChiA were active. This was not the case, because chiA expression is usually si- lenced by the nucleotide-structuring protein $\mathrm{H}-\mathrm{NS}$ [Francetic et al., 2000a]. In contrast, ChiP expression is controlled by the small RNA ChiX but, expression of the $c h b$ transcript relives $c h i P$ inhibition by functioning as trap-RNA [Figueroa-Bossi et al., 2009; Rasmussen et al., 2009]. Thus, expression of ChiA in the periplasm should allow growth on chitooligomers (e.g., pentaacetyl-chitopentaose), which are transported through $\mathrm{ChiP}$ into the periplasm. Cleavage by ChiA releases diacetyl-chitobiose and triacetyl-chitotriose, which can be taken up by the ChbCBA-PTS and metabolized by ChbG and ChbF. To test this, ChiA was artificially expressed in E. coli BL21 (DE3) from plasmid pET22bchiA in an IPTG-inducible manner. Compared to E. coli K12 wt during growth in M9 CAA (to avoid carbon catabolite repression) minimal medium, the expression of ChiA clearly restored the availability to utilize pentaacetyl-chitopentaose (Fig. 10d). To exclude that E. coli 


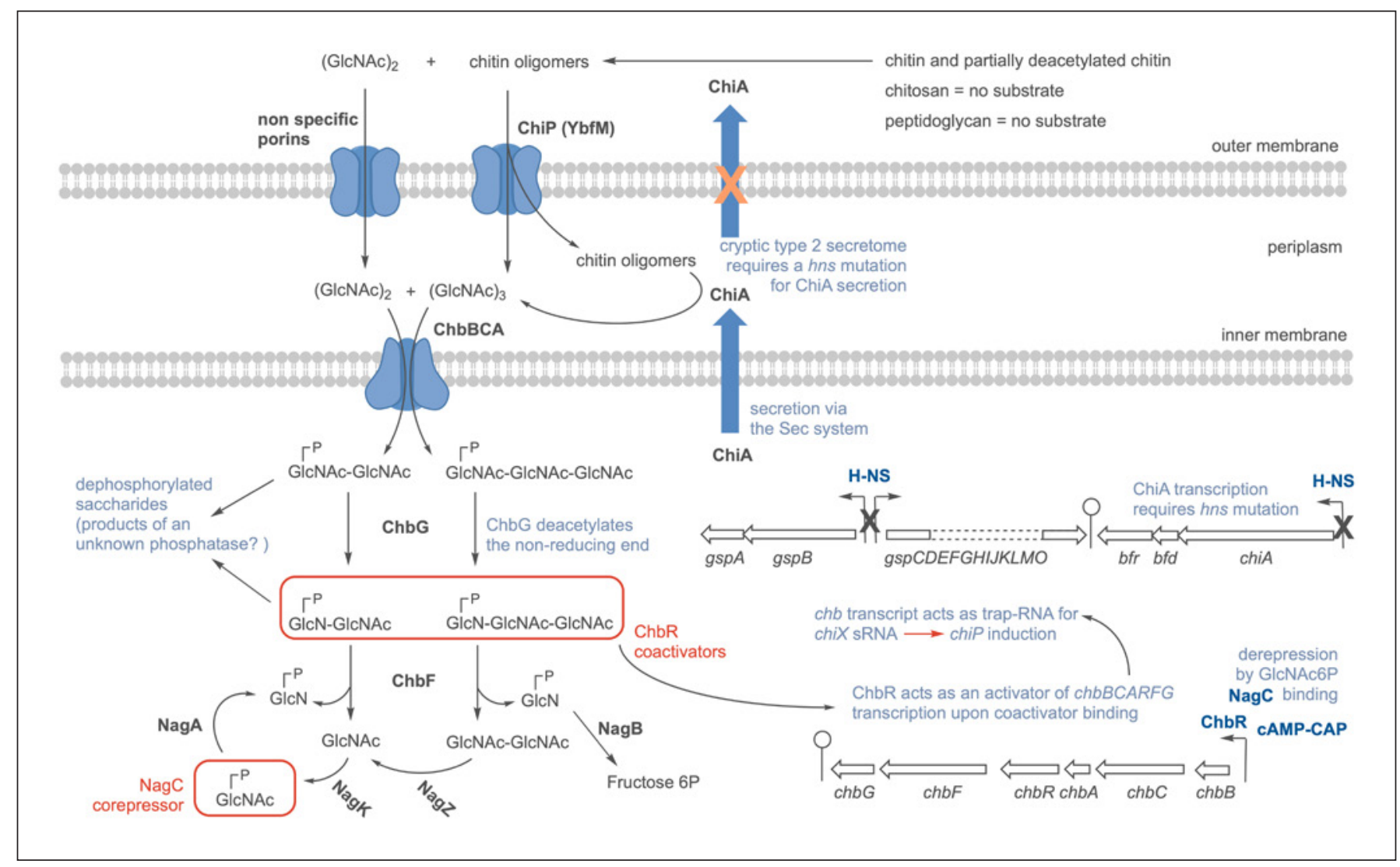

Fig. 11. Overview of the chitin, chitin oligomer, triacetyl-chitotriose, and diacetyl-chitobiose metabolism of E. coli and its regulation. Chitin and partially acetylated chitosan is cleaved by the chitinase ChiA, yielding transiently chitin oligomers and as final products triacetyl-chitotriose $\left(\mathrm{GlcNAc}_{3}\right)$ and diacetyl-chitobiose $\left(\mathrm{GlcNAc}_{2}\right)$. In E. coli $\mathrm{K} 12$, however, ChiA expression is repressed (silenced) by the nucleoid-structuring protein $\mathrm{H}-\mathrm{NS}$ [Francetic et al., 2000a]. ChiA is exported into the periplasm via Sec secretion, provided it is expressed either artificially or in a $h n s$ mutant. In a hns strain, ChiA also gets partially secreted into the medium via activation of the cryptic type 2 secretome [Francetic et al., 2000b]. Diacetyl-chitobiose is taken up into the periplasm by nonspecific porins, whereas triacetyl-chitotriose and short-chain chitin oligomers pass through the membrane by a facilitated diffusion process mediated by the chitoporin ChiP [Soysa and Suginta, 2016]. If present in the periplasm, ChiA can cleave the chitin oligomers to diacetyl-chitobiose and triacetyl-chitotriose. E. coli grows on diacetyl-chitobiose and triacetyl-chitotriose via the expression of the chb operon (chbBCARFG). The phosphotransferase system (PTS)

BL21 (DE3) expressed endogenous ChiA we monitored its growth on penta-acetyl-chitopentaose and observed the same growth pattern as that of E. coli K12 wt (data not shown). This clearly shows that $E$. coli has the potential to utilize chitin oligosaccharides in a ChiA-dependent manner. However, since E. coli ChiA expres- transporter ChbBCA facilitates transport of both saccharides across the inner membrane and their concomitant phosphorylation at the non-reducing end. Subsequently, the phosphorylated saccharides are deacetylated at the non-reducing end by ChbG yielding monoacetyl-chitobiose-P (GlcN6P-GlcNAc) and diacetyl-chitotriose-P (GlcN6P-GlcNAc-GlcNAc). These two products also act as coactivators for ChbR (framed in red), resulting in increased transcription of the $c h b$ operon. They are cleaved by $\mathrm{ChbF}$ and further processed by the exo-glucosaminidase NagZ [Litzinger et al., 2010a, b] and the $\mathrm{N}$-acetyl-D-glucosamine kinase NagK [Uehara and Park, 2004]. The $N$-acetylglucosamine repressor $\mathrm{NagC}$, which binds the chb promoter thereby inhibiting transcription, is released from the promoter by binding to GlcNAc6P (framed in red) [Plumbridge and Pellegrini, 2004]. Expression of the chb operon is also subject to catabolite repression (i.e., activated by cAMP-CAP). GlcNAc6P is degraded by the GlcNAc6P deacetylase NagA and GlcN-6P deaminase NagB to yield fructose $6 \mathrm{P}$ thereby entering glycolysis.

sion and secretion is silenced, the bacterium either relies on scavenging chitinase end products from other organisms or, if there is enough evolutionary pressure, selection against $\mathrm{H}-\mathrm{NS}$ repression of $\mathrm{ChiA}$ and the type 2 secretome might occur. 


\section{Conclusion}

In this study we refined the understanding of the catabolic pathways of diacetyl-chitobiose (GlcNAc-GlcNAc) and triacetyl-chitotriose (GlcNAc-GlcNAc-GlcNAc) in E. coli, as summarized in Figure 11. Catabolism of the saccharides involves phosphorylation via the ChbBCA PTS transporter, as shown previously [Keyhani et al., 2000a, b, c], and subsequent deacetylation via the monodeacetylase ChbG, which specifically removes the $N$-acetyl group from non-reducing end terminal GlcNAc6P entities, as shown in this study. Since activation of the chb promoter by the transcriptional regulator ChbR depends on the ChbG activity [Verma and Mahadevan, 2012], the correction of the function of ChbG indirectly identified GlcN6PGlcNAc and GlcN6P-GlcNAc-GlcNAc as the inducers of the $c h b$ operon, which upon binding to $\mathrm{ChbR}$ activate $c h$ $b B C A R F G$ transcription. These saccharides were found to accumulate in a $\triangle c h b F$ strain, thus constituting the natural substrates of ChbF. Intriguingly, ChbF failed to cleave GlcNAc6P-GlcNAc, because the $N$-acetyl group of GlcNAc6P-glycosides sterically interferes with amino acids in the active site pocket and thus prevents binding to $\mathrm{ChbF}$, as shown by structural modeling. Possibly, the unusual redox/elimination-type mechanism of ChbF, belonging to family 4 of Cazy glycosidases (URL: http:// www.cazy.org/GH4.html) [Henrissat, 1991; Davies and Henrissat, 1995; Yip and Withers, 2012], excludes GlcNAc6P-glycosides as suitable substrates. Accumulation of non-phosphorylated saccharides in the $c h b F$ mutant indicates that an unidentified phosphatase is active intracellularly that cleaves the phosphorylated 6P-glycosides. We assume that the dephosphorylation occurs due to the accumulation of high concentrations of phosphorylated saccharides and, thus, likely constitutes a detoxification mechanism counteracting sugar phosphate stress. The identity of the phosphatase is so far unclear; however, it could be identical to the phosphatase YigL, which has been reported to relieve glucose-phosphate stress in $E$. coli [Kuznetsova et al., 2006; Sun and Vanderpool, 2013].

The reason for this particular catabolic pathway in $E$. coli is unclear, but may be linked to the function of the chitinase ChiA of E. coli. We showed that the chitinase ChiA specifically cleaves chitin as well as acetylated chitosan, yielding diacetyl-chitobiose/triacetyl-chitotriose and GlcN-GlcNAc-GlcNAc, respectively, but does not cleave intact or peptide-free, denuded peptidoglycan as well as chitosan. Chitin in nature barely occurs as a fully acetylated polymer. Since ChiA is able to cleave GlcNAcbeta-1,4-GlcN bonds, it thus generates saccharides that upon uptake and phosphorylation by the ChbBCA PTS directly act as inducers of the chb operon. A still open question is why ChiA expression is silenced in E. coli. It is appealing to assume that $E$. coli laboratory strains have adapted to the use of LB- or similar media made up of yeast extract, which contains diacetyl-chitobiose and triacetyl-chitotriose but lacks chitooligomers; thus, ChiA is dispensable under these condition. It could be interesting to screen for undomesticated E. coli strains that lack a silent ChiA system and hence retain their ability to grow on polymeric chitin.

\section{Experimental Procedures}

\section{Bacterial Strains and Growth Conditions}

The bacterial strains used in this study are listed in online supplementary Table S1. B. subtilis 168 wild-type and E. coli K12 were used for the isolation of peptidoglycan. Bacteria were cultured at $37^{\circ} \mathrm{C}$ in lysogeny broth (LB broth Lennox, Carl Roth) with continuous shaking at $140 \mathrm{rpm}$. Overnight cultures $(\sim 16 \mathrm{~h})$ were used to inoculate fresh LB medium. Cells were harvested by centrifugation $\left(3,000 \times g, 20 \mathrm{~min}, 4^{\circ} \mathrm{C}\right)$. E. coli BL21 (DE3) cells (New England Biolabs) used to heterologously express recombinant enzymes were grown in LB medium supplemented with $50 \mu \mathrm{g} / \mathrm{mL}$ kanamycin or $100 \mu \mathrm{g} / \mathrm{mL}$ ampicillin depending on the expression plasmid until $\mathrm{OD}_{600} 0.7$ was reached, followed by induction with $1 \mathrm{~mm}$ IPTG and further propagation for $3 \mathrm{~h}$. Cells were harvested by centrifugation $\left(3,000 \times g, 20 \mathrm{~min}, 4^{\circ} \mathrm{C}\right)$ and used for the purification of recombinant proteins. For the extraction of accumulation products E. coli $\Delta$ chbG::kan, $\Delta$ chbF::kan, and E. coli $\Delta$ chbG::kan pQE32-chbBCA were grown at $37^{\circ} \mathrm{C}$ o/n in $5 \mathrm{~mL} \mathrm{M} 9$ minimal medium [Harwood and Cutting, 1990] with $6 \mathrm{~g} / \mathrm{L}$ casamino acids (CAA) instead of glucose and $2 \mathrm{~mm}$ diacetyl-chitobiose. The growth curves were conducted in a Tecan plate reader at $37^{\circ} \mathrm{C}$ in 24-well plates filled with $400 \mu \mathrm{L}$ M9 minimal medium [Harwood and Cutting, 1990] either with $0.1 \%$ glucose or $0.1 \%$ CAA and $0.1 \%$ of test substrate (diacetyl-chitobiose, triacetyl-chitotriose or penta-acetyl-chitopentaose; obtained from Carbosynth).

\section{Construction of Plasmids and Purification of Recombinant}

Enzymes

For the overexpression and purification of the enzymes used in this work, E. coli K12 ChbF (JW1723) and ChiA (JW3300), B. subtilis 168 Csn (BSU26890), NagZ (BSU01660), and CwlC (BSU17410), and S. aureus USA300 Atl (Glc) (ABD22514.1), the corresponding genes were amplified by PCR using the respective genomic DNA and primers listed in online supplementary Table S1. Prior to its insertion in pET28a, the NcoI site in $c w l$ was deleted. The PCR products were purified (GeneJET purification kit and GeneRuler, 1-kb marker, Thermo Fisher Scientific). PCR product and vector (specified in primer name in online suppl. Table S1) were digested with restriction enzymes (specified in primer name in online suppl. Table S1; New England Biolabs) and ligated with T4 DNA ligase (Thermo Fisher Scientific). Chemically competent $E$. coli DH5a cells were transformed using the constructed plasmids and selected for growth on the respective 
antibiotic. From positive clones plasmids were purified and checked by sequencing.

The positive plasmids were used to transform E. coli BL21 (DE3) cells, which were grown as described above and lysed in a French pressure cell. Recombinant His-tagged enzymes were purified by $\mathrm{Ni}^{2+}$-affinity chromatography using a 1-mL HisTrap column (GE Healthcare) followed by size exclusion chromatography on a HiLoad 16/60 Superdex 200 pg column (GE Healthcare) and purity was checked with a $12 \%$ SDS-PAGE. From a 1-L culture 3.6 $\mathrm{mg} \mathrm{ChbF}, 1.75 \mathrm{mg} \mathrm{CwlC}$, and $12.5 \mathrm{mg}$ Csn were obtained. The enzymes were stored at $-20^{\circ} \mathrm{C}$ in $20 \mathrm{mM} \mathrm{Na}_{2} \mathrm{HPO}_{4}$ buffer $(500 \mathrm{mM}$ $\mathrm{NaCl}, \mathrm{pH}$ 7.6). Gibson Assembly was used for the construction of a PTS transporter mutant in E. coli $\Delta c h b G:: k a n$. The corresponding gene sections for $c h b B C A$ were amplified by PCR from $E$. coli K12 genomic DNA using the appropriate primers (online suppl. Table S1). The purified PCR product was cloned via Gibson Assembly into pQE32, which was linearized via digestion with BseRI and HindIII. Chemically competent E. coli $\Delta$ chbG::kan cells were transformed with $\mathrm{pQE} 32-\mathrm{chbBCA}$. Positive clones selected by antibiotics were confirmed by sequencing.

\section{Preparation of Peptidoglycan}

For the preparation of $B$. subtilis 168 cell walls $2 \mathrm{~L}$ of culture (exponential growth phase, $\mathrm{OD}_{600}=1.0$ ) were harvested and resuspended in $30 \mathrm{~mL}$ piperazine-acetate buffer $(50 \mathrm{mM}, \mathrm{pH}$ 6) with 12 $\mathrm{U}$ proteinase $\mathrm{K}$ and boiled for $1 \mathrm{~h}$. The cytosolic fractions were removed by centrifugation $\left(3,000 \times \mathrm{g}, 15 \mathrm{~min}, 4^{\circ} \mathrm{C}\right)$. The pellet was resuspended in $6 \mathrm{~mL}$ buffer $(10 \mathrm{mM}$ Tris, $10 \mathrm{mM} \mathrm{NaCl}, 320 \mathrm{mM}$ imidazole, adjusted to $\mathrm{pH} 7.0$ with $\mathrm{HCl}$ ) and $600 \mu \mathrm{g}$ a-amylase, 250 U RNase A, $120 \mathrm{U}$ DNase I, and $50 \mathrm{mM} \mathrm{MgSO}_{4}$ were added. The sample was incubated at $37^{\circ} \mathrm{C}$ for $2 \mathrm{~h}$ while shaking, $12 \mathrm{U}$ Proteinase $\mathrm{K}$ was added, and the incubation continued for $1 \mathrm{~h}$. $4 \%$ SDS solution was added 1:1 and the mixture was boiled for $1 \mathrm{~h}$. SDS was removed by repeated ultracentrifugation steps (20 times at 140,000 $\times \mathrm{g}, 30 \mathrm{~min}, 40^{\circ} \mathrm{C}$ ) and suspension in $\mathrm{H}_{2} \mathrm{O}_{\text {bidistilled }}$ as well as dialysis

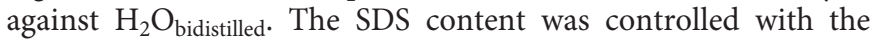
methylene blue assay described earlier [Hayashi, 1975]. The cell wall preparation was dried in a vacuum concentrator. Wall teichoic acids were removed by incubation with $1 \mathrm{M} \mathrm{HCl}$ for $4 \mathrm{~h}$ at $37^{\circ} \mathrm{C}$.

For the preparation of $E$. coli $\mathrm{K} 12$ cell walls $2 \mathrm{~L}$ of culture (exponential growth phase, $\left.\mathrm{OD}_{600}=1.0\right)$ were harvested and resuspended in $90 \mathrm{~mL}$ ice-cold $\mathrm{H}_{2} \mathrm{O}_{\text {bidistilled }}$ (to prevent peptidoglycan autolysis activity). Boiling SDS solution (8\%) was added dropwise until a final volume of $100 \mathrm{~mL}$ and boiled for $1 \mathrm{~h}$ further while stirring constantly. The SDS was removed as described for B. subtilis cell wall. After resuspending the pellet again in $20 \mathrm{~mL} \mathrm{H}_{2} \mathrm{O}_{\text {bidistilled, }}$ $300 \mu \mathrm{L}$ a-amylase stock solution $(12 \mathrm{mg}$ a-amylase $+0.5 \mathrm{~mL}$ $\mathrm{H}_{2} \mathrm{O}_{\text {bidistilled }}+0.7 \mathrm{~mL} 10 \mathrm{~mm}$ Tris- $\mathrm{HCl} \mathrm{pH} \mathrm{7)} \mathrm{were} \mathrm{added} \mathrm{and} \mathrm{in-}$ cubated at $37^{\circ} \mathrm{C}$ under constant shaking for $2 \mathrm{~h}$. Pronase solution (100 mg pronase $+10 \mathrm{~mL} 10 \mathrm{mM}$ Tris- $\mathrm{HCl}$ buffer $\mathrm{pH}$ 7) was preincubated at $60^{\circ} \mathrm{C}$ for $2 \mathrm{~h} .500 \mu \mathrm{L}$ pronase solution was added to the sample and incubation was continued for $2 \mathrm{~h}$ further. The sample was boiled for $1 \mathrm{~h}$ while stirring followed by one ultracentrifugation step. The cell wall preparation was dried in a vacuum concentrator.

\section{Preparation of Colloidal Chitin}

For the preparation of colloidal chitin chitosan $(<3 \mathrm{kDa}$; obtained from Carbosynth) was acetylated with acetic anhydride. 20 $\mathrm{mL} 1 \mathrm{M}$ sodium bicarbonate (freshly prepared) were added to 500 mg chitosan and mixed by swirling until a brown-colored foam developed. $0.5 \mathrm{~mL}$ of $>99 \%$ acetic anhydride were added dropwise while mixing slowly, followed by incubation for $20 \mathrm{~min}$ at room temperature. The solution was precipitated by centrifugation $(1,500 \times g)$ and the pellet was resuspended again in $20 \mathrm{~mL} 1 \mathrm{M}$ sodium bicarbonate with $0.5 \mathrm{~mL}$ acetic anhydride and further incubated for $20 \mathrm{~min}$. The reaction was boiled for $10 \mathrm{~min}$ and washed with phosphate buffered saline until $\mathrm{pH} 7$ was attained. The colloidal chitin was stored at $4^{\circ} \mathrm{C}$.

\section{Enzymatic Reactions}

For the digestion of E. coli and B. subtilis peptidoglycan, colloidal chitin, and chitosan oligomers, $0.2 \mathrm{mg}$ substrate were used. The following buffers were used for the enzymes: $0.1 \mathrm{M}$ potassium phosphate $\mathrm{pH} 8$ for Atl and $\mathrm{CwlC}$; $0.1 \mathrm{M}$ sodium phosphate $\mathrm{pH} 7.2$ for ChiA, chitinase from S. griseus, NagZ and ChbF; 0.1 M potassium phosphate $\mathrm{pH} 6$ for mutanolysin; $0.05 \mathrm{M}$ sodium acetate $\mathrm{pH}$ 5.7 for Csn. In each case $5 \mu \mathrm{g}$ of enzyme were used for one preparation and all reactions took place at $37^{\circ} \mathrm{C}$. Peptidoglycan preparations were incubated for $1 \mathrm{~h}$, colloidal chitin, chitosan, and cytosolic fractions for $2 \mathrm{~h}$. The digestion volume was $50 \mu \mathrm{L}$ total, except for the pre-digestion of peptidoglycan by $\mathrm{CwlC}$, which had a volume of $25 \mu \mathrm{L}$. After CwlC pre-treatment the $\mathrm{pH}$ was adjusted to the corresponding $\mathrm{pH}$ value of the subsequent enzyme with $0.1 \mathrm{M}$ potassium dihydrogen phosphate and the volume was adjusted to $50 \mu \mathrm{L}$. After each digestion the enzymes were heat-inactivated at $95^{\circ} \mathrm{C}$ for $10 \mathrm{~min}$.

\section{Generation of Cytosolic Fractions}

A 5-mL culture was harvested by centrifugation at $16,000 \times g$ for $10 \mathrm{~min}$ and was washed twice in $1 \mathrm{~mL}$ Tris- $\mathrm{HCl}$ ( $\mathrm{pH} 7.6,10$ $\mathrm{mM}$ ) buffer. The cells were resuspended in $200 \mu \mathrm{L}$ buffer and incubated for $10 \mathrm{~min}$ at $95^{\circ} \mathrm{C}$ followed by a further centrifugation step. The supernatant was removed by pipetting and mixed with $800 \mu \mathrm{L}$ acetone. After a further centrifugation step the supernatant was removed and dried under vacuum at $37^{\circ} \mathrm{C}$. Samples were dissolved in $30 \mu \mathrm{L} \mathrm{H} \mathrm{O}_{2} \mathrm{O}_{\text {bidistilled }}$ for LC-MS analysis [Gisin et al., 2013].

\section{Reduction of Samples with $\mathrm{NaBH}_{4}$}

Samples were reduced with sodium borohydride as described earlier [Schaub and Dillard, 2017; Kluj et al., 2018]. To prepare the reducing solution, $500 \mu \mathrm{L}$ of $0.5 \mathrm{M}$ borate buffer ( $\mathrm{pH}$ 9) was added to $5 \mathrm{mg}$ sodium borohydride. Samples dissolved in $30 \mu \mathrm{L}$ $\mathrm{H}_{2} \mathrm{O}_{\text {bidistilled }}$ were added to $30 \mu \mathrm{L}$ reducing solution and incubated for $20 \mathrm{~min}$ at room temperature. By the addition of $10 \mu \mathrm{L} 8.5 \%$ phosphoric acid the $\mathrm{pH}$ was adjusted to 3-4 before the samples were dried in a vacuum concentrator at $37^{\circ} \mathrm{C}$ and dissolved in 30 $\mu \mathrm{L} \mathrm{H}_{2} \mathrm{O}_{\text {bidistilled }}$ for LC-MS analysis.

\section{Analysis of Reaction Products by LC-MS}

Sample analysis was conducted using an electrospray ionization-time of flight (ESI-TOF) mass spectrometer (MicrOTOF II; Bruker Daltonics), operated in positive ion-mode that was connected to an UltiMate 3000 high-performance liquid chromatography (HPLC) system (Dionex). For HPLC-MS analysis $5 \mu \mathrm{L}$ of the sample supernatant were injected into a Gemini C18 column (150 $\times 4.6 \mathrm{~mm}, 5 \mu \mathrm{m}, 110 \AA$, Phenomenex). A 45 -min program at a flow rate of $0.2 \mathrm{~mL} / \mathrm{min}$ was used to separate the compounds as previously described [Gisin et al., 2013]. An Orbitrap Elite mass spectrometer (Thermo Scientific) was used to record higher energy collisional dissociation (HCD) MS/MS spectra. 


\section{Acknowledgement}

We acknowledge Marina Borisova, Tim Teufel, and Amanda Duckworth for providing plasmids and proteins as well as Johannes Madlung and Boris Macek for valuable expertise with MS/ MS measurements.

\section{Statement of Ethics}

Ethics approval was not required.

\section{Conflict of Interest Statement}

The authors have no conflicts of interest to declare.

\section{Funding Sources}

C.M. acknowledges funding by the ministry of science, research and art of the state Baden-Württemberg (program glycobiology and glycobiotechnology) and by the Deutsche Forschungsgemeinschaft (DFG, German Research Foundation), grants SFB766, Project-ID 398967434 - TRR 261 and Project-ID 174858087 - GRK1708. This work was further supported by the DFG-funded Cluster of Excellence EXC 2124 Controlling Microbes to Fight Infections.

\section{Author Contributions}

A.W. conducted the experiments. S.F. provided the plasmid pQE32-ChbBCA. C.M. formulated the original problem and provided guidance throughout the study. C.M. and A.W. designed the experiments and developed the methodology. C.M., S.F., and A.W. wrote the manuscript.

\section{References}

Adams DW, Stutzmann S, Stoudmann C, Blokesch M. DNA-uptake pili of Vibrio cholerae are required for chitin colonization and capable of kin recognition via sequence-specific self-interaction. Nat Microbiol. 2019 Sep; $4(9): 1545-57$

Blokesch M. Chitin colonization, chitin degradation and chitin-induced natural competence of Vibrio cholerae are subject to catabolite repression. Environ Microbiol. 2012 Aug;14(8) 1898-912.

Davies G, Henrissat B. Structures and mechanisms of glycosyl hydrolases. Structure. 1995 3(9):853-9.

Figueroa-Bossi N, Valentini M, Malleret L, Fiorini F, Bossi L. Caught at its own game: regulatory small RNA inactivated by an inducible transcript mimicking its target. Genes Dev. 2009 Sep 1;23(17):2004-15.

Francetic O, Badaut C, Rimsky S, Pugsley AP. The ChiA (YheB) protein of Escherichia coli K-12 is an endochitinase whose gene is negatively controlled by the nucleoid-structuring protein H-NS. Mol Microbiol. 2000a Mar;35(6): 1506-17.

Francetic O, Belin D, Badaut C, Pugsley AP. Expression of the endogenous type II secretion pathway in Escherichia coli leads to chitinase secretion. EMBO J. 2000b Dec 15;19(24): 6697-703.

Gisin J, Schneider A, Nägele B, Borisova M, Mayer C. A cell wall recycling shortcut that bypasses peptidoglycan de novo biosynthesis. Nat Chem Biol. 2013 Aug;9(8):491-3.

Hamid R, Khan MA, Ahmad M, Ahmad MM, Abdin MZ, Musarrat J, et al. Chitinases: An update. J Pharm Bioallied Sci. 2013 Jan;5(1):219.

Harwood CR, Cutting SM. Chemically defined growth media and supplements. Wiley, Chichester, UK; 1990.
Hayashi K. A rapid determination of sodium dodecyl sulfate with methylene blue. Anal Biochem. 1975 Aug;67(2):503-6.

Henrissat B. A classification of glycosyl hydrolases based on amino acid sequence similarities. Biochem J. 1991;280 ( Pt 2):309-16.

Itoh T, Kimoto $\mathrm{H}$. Bacterial chitinase system as a model of chitin biodegradation. Adv Exp Med Biol. 2019;1142:131-51.

Keyhani NO, Bacia K, Roseman S. The transport/ phosphorylation of N,N'-diacetylchitobiose in Escherichia coli. Characterization of phospho-IIBChb and of a potential transition state analogue in the phosphotransfer reaction between the proteins IIAChb and IIBChb. J Biol Chem. 2000a;275(42):33102-9.

Keyhani NO, Boudker O, Roseman S. Isolation and characterization of IIAChb, a soluble protein of the enzyme II complex required for the transport/phosphorylation of N, N'-diacetylchitobiose in Escherichia coli. J Biol Chem. 2000b;275(42):33091-101.

Keyhani NO, Roseman S. Wild-type Escherichia coli grows on the chitin disaccharide, N,N'diacetylchitobiose, by expressing the cel operon. Proc Natl Acad Sci USA. 1997;94(26): 14367-71.

Keyhani NO, Wang LX, Lee YC, Roseman S. The chitin disaccharide, N,N'-diacetylchitobiose, is catabolized by Escherichia coli and is transported/phosphorylated by the phosphoenolpyruvate:glucose phosphotransferase system. J Biol Chem. 2000c;275(42):33084-90.

Kluj RM, Ebner P, Adamek M, Ziemert N, Mayer C, Borisova M. Recovery of the peptidoglycan turnover product released by the autolysin Atl in Staphylococcus aureus involves the phosphotransferase system transporter MurP and the novel 6-phospho- $\mathrm{N}$-acetylmuramidase MupG. Front. Microbiol. 2018 Nov 16; 9(2725).
Kuznetsova E, Proudfoot M, Gonzalez CF, Brown $\mathrm{G}$, Omelchenko MV, Borozan I, et al. Genome-wide analysis of substrate specificities of the Escherichia coli haloacid dehalogenaselike phosphatase family. J Biol Chem. 2006 Nov 24;281(47):36149-61.

Litzinger S, Duckworth A, Nitzsche K, Risinger C, Wittmann V, Mayer C. Muropeptide rescue in Bacillus subtilis involves sequential hydrolysis by beta- $\mathrm{N}$-acetylglucosaminidase and $\mathrm{N}$ acetylmuramyl-L-alanine amidase. J Bacteriol. 2010a Jun;192(12):3132-43.

Litzinger S, Fischer S, Polzer P, Diederichs K, Welte W, Mayer C. Structural and kinetic analysis of Bacillus subtilis N-acetylglucosaminidase reveals a unique Asp-His dyad mechanism. J Biol Chem. 2010b Nov 12; 285(46):35675-84.

Müller M, Calvert M, Hottmann I, Kluj RM, Teufel T, Balbuchta K, et al. The exo- $\beta-\mathrm{N}$ acetylmuramidase NamZ from Bacillus subtilis is the founding member of a family of exolytic peptidoglycan hexosaminidases. $J$ Biol Chem. 2021. http://dx.doi.org/10.1016/j. jbc.2021.100519. In press.

Oshida T, Sugai M, Komatsuzawa H, Hong YM, Suginaka H, Tomasz A. A Staphylococcus aureus autolysin that has an $\mathrm{N}$-acetylmuramoyl$\mathrm{L}$-alanine amidase domain and an endo- $\beta-\mathrm{N}$ acetylglucosaminidase domain: cloning, sequence analysis, and characterization. Proc Natl Acad Sci USA. 1995 Jan 3;92(1):285-9.

Parker LL, Hall BG. Mechanisms of activation of the cryptic cel operon of Escherichia coli K12. Genetics. 1990;124(3):473-82.

Plumbridge J, Pellegrini O. Expression of the chitobiose operon of Escherichia coli is regulated by three transcription factors: NagC, ChbR and CAP. Mol Microbiol. 2004 Apr;52(2): 437-49. 
Rajan SS, Yang X, Collart F, Yip VLY, Withers SG, Varrot A, et al. Novel Catalytic Mechanism of Glycoside Hydrolysis Based on the Structure of an NAD+/Mn2+-Dependent Phospho- $\alpha$ Glucosidase from Bacillus subtilis. Structure. 2004 Sep;12(9):1619-29.

Rasmussen AA, Johansen J, Nielsen JS, Overgaard M, Kallipolitis B, Valentin-Hansen P. A conserved small RNA promotes silencing of the outer membrane protein YbfM. Mol Microbiol. 2009 May;72(3):566-77.

Rau A, Hogg T, Marquardt R, Hilgenfeld R. A new lysozyme fold. Crystal structure of the muramidase from Streptomyces coelicolor at $1.65 \AA$ resolution. J Biol Chem. 2001 Aug 24; 276(34):31994-9.

Rivas LA, Parro V, Moreno-Paz M, Mellado RP. The Bacillus subtilis $168 \mathrm{csn}$ gene encodes a chitosanase with similar properties to a Streptomyces enzyme. Microbiology (Reading, Engl). 2000 Nov;146(Pt 11):2929-36.

Schaub RE, Dillard JP. Digestion of peptidoglycan and analysis of soluble fragments. Bio Protoc. 2017 Aug 5;7(15).

Shida T, Hattori H, Ise F, Sekiguchi J. Overexpression, purification, and characterization of $\mathrm{Ba}$ cillus subtilis $\mathrm{N}$-acetylmuramoyl-L-alanine amidase CwlC. Biosci Biotechnol Biochem. 2000 Jul;64(7):1522-5.
Soysa HS, Suginta W. Identification and functional characterization of a novel OprD-like chitin uptake channel in non-chitinolytic bacteria. J Biol Chem. 2016 Jun 24;291(26): 13622-33.

Sun Y, Vanderpool CK. Physiological consequences of multiple-target regulation by the small RNA SgrS in Escherichia coli. J Bacteriol. 2013 Nov; 195(21):4804-15.

Tharanathan RN, Kittur FS. Chitin--the undisputed biomolecule of great potential. Crit Rev Food Sci Nutr. 2003;43(1):61-87.

Thompson J, Ruvinov SB, Freedberg DI, Hall BG. Cellobiose-6-phosphate hydrolase (CelF) of Escherichia coli: characterization and assignment to the unusual family 4 of glycosylhydrolases. J Bacteriol. 1999;181(23):7339-45.

Uehara T, Park JT. The N-acetyl-D-glucosamine kinase of Escherichia coli and its role in murein recycling. J Bacteriol. 2004 Nov; 186(21): 7273-9.

Verma SC, Mahadevan S. The chbG gene of the chitobiose (chb) operon of Escherichia coli encodes a chitooligosaccharide deacetylase. J Bacteriol. 2012 Sep;194(18):4959-71.

Wang SL, Chang WT. Purification and characterization of two bifunctional chitinases/lysozymes extracellularly produced by Pseudomonas aeruginosa $\mathrm{K}-187$ in a shrimp and crab shell powder medium. Appl Environ Microbiol. 1997 Feb;63(2):380-6.
Wucher BR, Bartlett TM, Hoyos M, Papenfort K Persat A, Nadell CD. Vibrio cholerae filamentation promotes chitin surface attachment at the expense of competition in biofilms. Proc Natl Acad Sci USA. 2019 Jul 9;116(28):1421621.

Yip VL, Thompson J, Withers SG. Mechanism of GlvA from Bacillus subtilis: a detailed kinetic analysis of a 6-phospho- $\alpha$-glucosidase from glycoside hydrolase family 4 . Biochemistry. 2007 Aug 28;46(34):9840-52.

Yip VL, Varrot A, Davies GJ, Rajan SS, Yang X, Thompson J, et al. An unusual mechanism of glycoside hydrolysis involving redox and elimination steps by a family 4 beta-glycosidase from Thermotoga maritima. J Am Chem Soc. 2004 Jul 14;126(27):8354-5.

Yip VL, Withers SG. Breakdown of oligosaccharides by the process of elimination. Curr Opin Chem Biol. 2006a Apr;10(2):147-55.

Yip VL, Withers SG. Mechanistic analysis of the unusual redox-elimination sequence employed by Thermotoga maritima BglT: a 6 -phospho- $\beta$-glucosidase from glycoside hydrolase family 4 . Biochemistry. 2006b Jan 17; 45(2):571-80.

Yip VL, Withers SG. Identification of Tyr241 as a key catalytic base in the family 4 glycoside hydrolase BglT from Thermotoga maritima. Biochemistry. 2012 Oct 23;51(42):8464-74 